\title{
Enhancing CAR T cell persistence through ICOS and 4-1BB costimulation
}

\author{
Sonia Guedan, ${ }^{1}$ Avery D. Posey Jr., ${ }^{1}$ Carolyn Shaw, ${ }^{1}$ Anna Wing, ${ }^{1}$ Tong Da, ${ }^{1}$ Prachi R. Patel, ${ }^{1}$ \\ Shannon E. McGettigan, ${ }^{1}$ Victoria Casado-Medrano,, ${ }^{2}$ Omkar U. Kawalekar, ${ }^{1}$ Mireia Uribe-Herranz, ${ }^{3}$ \\ Decheng Song, ${ }^{1}$ J. Joseph Melenhorst, ${ }^{1}$ Simon F. Lacey, ${ }^{1}$ John Scholler, ${ }^{1}$ Brian Keith, ${ }^{1}$ Regina M. \\ Young, ${ }^{1}$ and Carl H. June ${ }^{1}$ \\ 'Center for Cellular Immunotherapies, Department of Pathology and Laboratory Medicine, 2Department of Systems \\ Pharmacology and Translational Therapeutics, and ${ }^{3}$ Department of Radiation Oncology, Perelman School of Medicine, \\ University of Pennsylvania, Philadelphia, Pennsylvania, USA.
}

Successful tumor eradication by chimeric antigen receptor-expressing (CAR-expressing) T lymphocytes depends on CAR T cell persistence and effector function. We hypothesized that $\mathrm{CD4}^{+}$and CD8 ${ }^{+} \mathrm{T}$ cells may exhibit distinct persistence and effector phenotypes, depending on the identity of specific intracellular signaling domains (ICDs) used to generate the CAR. First, we demonstrate that the ICOS ICD dramatically enhanced the in vivo persistence of CAR-expressing $\mathrm{CD4}^{+} \mathrm{T}$ cells that, in turn, increased the persistence of $\mathrm{CD}^{+} \mathrm{T}$ cells expressing either CD28- or 4-1BBbased CARs. These data indicate that persistence of $C D 8^{+} T$ cells was highly dependent on a helper effect provided by the ICD used to redirect CD4+ T cells. Second, we discovered that combining ICOS and 4-1BB ICDs in a third-generation CAR displayed superior antitumor effects and increased persistence in vivo. Interestingly, we found that the membrane-proximal ICD displayed a dominant effect over the distal domain in third-generation CARs. The optimal antitumor and persistence benefits observed in third-generation ICOSBBz CAR T cells required the ICOS ICD to be positioned proximal to the cell membrane and linked to the ICOS transmembrane domain. Thus, CARs with ICOS and 4-1BB ICD demonstrate increased efficacy in solid tumor models over our current 4-1BBbased CAR and are promising therapeutics for clinical testing.

Conflict of interest: The University of Pennsylvania has filed a patent application based on this work (application no. 14/376,038).

Submitted: August 18, 2017 Accepted: November 28, 2017 Published: January 11, 2018

\section{Reference information:} JCI Insight. 2018;3(1):e96976. https:// doi.org/10.1172/jci.insight.96976.

\section{Introduction}

Adoptive $\mathrm{T}$ cell immunotherapy, in which a patient's own $\mathrm{T}$ lymphocytes are engineered to express chimeric antigen receptors (CARs), has shown great promise in treating hematological malignancies (1). Multiple clinical trials have revealed that CARs directed against the CD19 cell surface protein can achieve long-term complete remissions in some patients with B cell malignancies (2-7). Despite these promising results, numerous technical and biological obstacles have limited the success of using CAR-modified $\mathrm{T}$ cells to treat solid tumors and have resulted in suboptimal responses in the setting of metastatic and/or recurrent disease (8-10).

Robust in vivo expansion and persistence of genetically modified $\mathrm{T}$ cells are considered critical predictors of durable clinical remissions in patients with hematologic malignancies $(6,11)$. It can be presumed that durable remissions for patients with solid tumors will also require significant in vivo expansion and long-term persistence of CAR T cells. Most CAR T cell clinical trials where lack of overall efficacy was observed have reported poor $\mathrm{T}$ cell persistence $(8,12,13)$. Several factors can influence the persistence of adoptively transferred $\mathrm{T}$ cells, including patient preconditioning, ex vivo culture conditions, development of $\mathrm{T}$ cell exhaustion, or host immune responses against the cellular infusion product $(7,9,12,13)$. Importantly, the molecular design of CARs is likely to strongly influence T cell expansion and persistence, and it is a focus of intensive research efforts $(14,15)$.

CARs commonly contain 3 modules: an extracellular target binding module, a transmembrane domain (TM domain), and an intracellular signaling domain (ICD) that transmits activation signals (15). TM domains are primarily considered a structural requirement, anchoring the CAR in the cell membrane, and are most commonly derived from molecules regulating $\mathrm{T}$ cell function, such as CD8 and CD28. The intracellular module typically consists of the $\mathrm{T}$ cell receptor $\mathrm{CD} 3 \zeta$ chain and 1 or more signaling domains from CD28, 4-1BB, OX40, CD27, or ICOS costimulatory proteins (14). CARs containing either CD28 or 4-1BB 
costimulatory domains have been the most widely used, to date, and both of them have yielded dramatic responses in clinical trials $(2-4,6,14)$. Several studies suggest that the CD28 intracellular domain stimulates greater CAR T cell functionality, whereas the 4-1BB intracellular domain promotes greater CAR $\mathrm{T}$ cell persistence. However, the mechanisms by which different TM and intracellular domains influence $\mathrm{T}$ cell expansion, function, and persistence are not yet fully understood.

Most of the recent clinical trials using CAR T cells have used cell products prepared from unselected "bulk" T cells. However, preclinical studies indicate that some $\mathrm{T}$ cell subtypes show distinct properties in vivo, such as enhanced proliferative capacity and increased antitumor effects $(16,17)$. CD4 ${ }^{+} \mathrm{T}$ cells provide cytokines and costimulation to the $\mathrm{CD} 8^{+}$populations, augmenting the priming, persistence, memory formation, and trafficking of cytotoxic effectors (18-20). Various CD4 ${ }^{+} \mathrm{T}$ cell subsets that differ in their capacities to proliferate and persist in vivo have been described, including Th1, Th2, Th9, Th17, and Tregs. However, $\mathrm{CD}^{+} \mathrm{T}$ cells are plastic, and the phenotype and function of these cells can evolve in vivo (16, 21, 22 ). Therefore, finding strategies to stabilize the phenotype of the infused cells to maintain their effector function and persistence would represent a significant advance in the field.

In recent work, we showed that incorporation of the ICOS intracellular domain into CARs augmented the effector function and in vivo persistence of Th17 polarized cells, compared with CARs with CD28 or 4-1BB intracellular domains (21). Here, we hypothesized that $\mathrm{CD}^{+}$and $\mathrm{CD} 8^{+} \mathrm{T}$ cell subsets require distinct costimulation signals for optimal persistence. We show that redirecting nonpolarized $\mathrm{CD}^{+} \mathrm{T}$ cells with an ICOS-based CAR significantly enhanced the persistence of $\mathrm{CD}^{+} \mathrm{T}$ cells expressing a 4-1BB- or CD28-based CAR. This observation led us to evaluate the efficacy of a third-generation CAR containing both ICOS and 4-1BB intracellular domains. Interestingly, incorporation of ICOS and 4-1BB in a CAR strongly enhanced both persistence and antitumor activity of CAR T cells, but only when ICOS was proximal to the cell membrane and linked to the ICOS TM domain. These results expand our understanding of CAR T cell responses, and provide a new strategy to optimize CAR $\mathrm{CD} 4^{+}$and $\mathrm{CD} 8^{+} \mathrm{T}$ cell expansion and persistence for superior antitumor function in patients with solid tumors.

\section{Results}

ICOS signaling drives $C D 4^{+}$T cells toward a Th1/Th17 phenotype. Our studies employed a CAR derived from a single chain variable fragment ( $\mathrm{scFv}$; SS1) that recognizes human mesothelin (unless otherwise indicated), which was fused to the T cell receptor- $\zeta(\mathrm{TCR}-\zeta)$ signal transduction domain $(\zeta)$ and 1 or more ICDs derived from ICOS, CD28, and 4-1BB (Figure 1A). CARs containing the CD28 ICD were linked to the CD28 TM domain, while CARs with a membrane-proximal ICOS ICD contained the ICOS TM domain (unless otherwise indicated). CARs with a membrane-proximal 4-1BB ICD contained the CD8- $\alpha$ TM domain, identical to the 4-1BB-based CARs used in the clinic by our group. A CAR that contained a truncated form of the TCR- $\varsigma$ intracellular domain was used as a control for signal transduction.

We first assessed the effects of individual ICDs on the effector functions of second-generation CAR $\mathrm{T}$ cells. All SS1-CARs were efficiently expressed on the surface of $\mathrm{T}$ cells, although expression of SS1CARs containing the ICOS costimulatory domain was slightly reduced compared with $28 \mathrm{z}$ and BBz CARs (Figure $1 \mathrm{~B}$ ). Both $\mathrm{CD}^{+}$and $\mathrm{CD} 8^{+} \mathrm{CAR} \mathrm{T}$ cells lysed non-small cell lung carcinoma cells (L55) efficiently compared with control $\mathrm{T}$ cells expressing a truncated $\zeta \mathrm{ICD}$, although $\mathrm{CD} 8^{+} \mathrm{T}$ cells killed tumor cells more rapidly than $\mathrm{CD}^{+} \mathrm{T}$ cells. No significant differences in cytotoxicity were observed between $\mathrm{T}$ cells expressing SS1 CARs with different signaling domains (Figure 1C).

We measured the effects of individual ICDs on cytokine release from $\mathrm{CD}^{+}$and $\mathrm{CD} 8^{+} \mathrm{CAR} \mathrm{T}$ cells following exposure to tumor cells that express mesothelin (Figure 1D). CD4 ${ }^{+}$ICOSz CAR T cells expressed high levels of IFN- $\gamma$, moderate levels of IL-17, and very low levels of Th2 cytokines, consistent with a predominantly Th1/Th17 phenotype (Figure 1, D and E). In contrast, CD4 ${ }^{+} 28 \mathrm{z}$ CAR T cells secreted greater levels of IL-2, TNF- $\alpha$, GM-CSF, IL-21, IL-4, IL-5, and IL-10 and very high levels of IL-13, consistent with a Th1/Th2 phenotype (Figure 1, D and E). Finally, CD4 ${ }^{+} \mathrm{BBz}$ CAR T cells expressed high levels of Th1 cytokines but low levels of most Th17 and Th2 cytokines - except for IL-13, which was expressed at high levels. Although no significant differences were observed on the levels of IFN- $\gamma$ released among groups (Supplemental Figure 1; supplemental material available online with this article; https:// doi.org/10.1172/jci.insight.96976DS1), the ratio of IL-2/IFN- $\gamma$ was significantly higher in the 28z CAR T cells when compared with ICOSz, while the ratio of IFN- $\gamma / \mathrm{TNF}-\alpha$ was significantly higher in ICOSz CAR $\mathrm{T}$ cells. Overall, these data indicate preferential expression of IFN- $\gamma$ versus most other cytokines analyzed 
A

CD8 $\alpha$ leader $] \mathrm{CD} 8 \alpha \mathrm{TM} \square \mathrm{ICOS}$ TM
$\mathrm{CD} 8 \alpha$ hinge

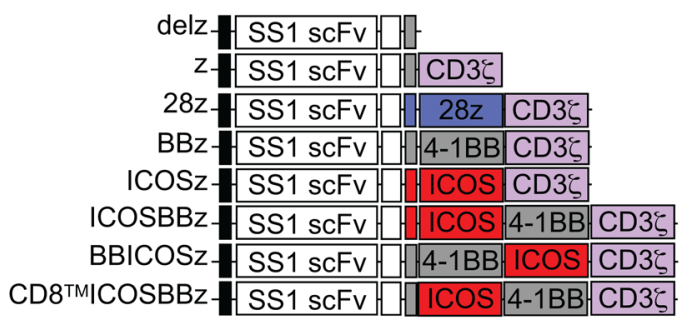

D
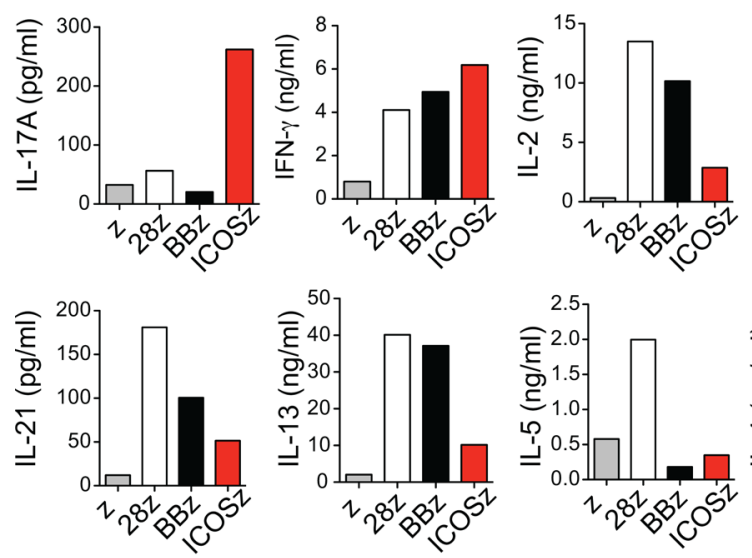

H
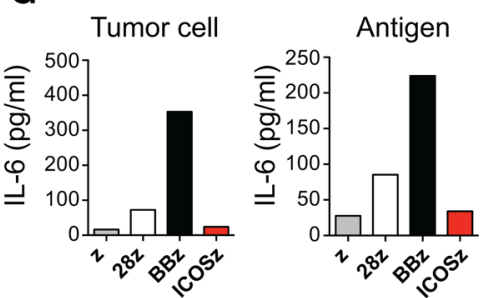
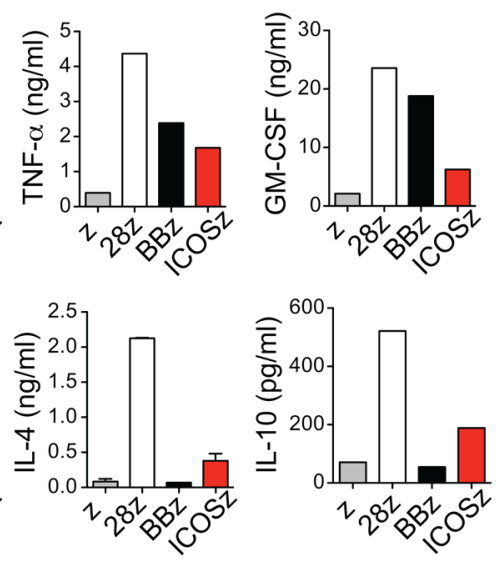

B
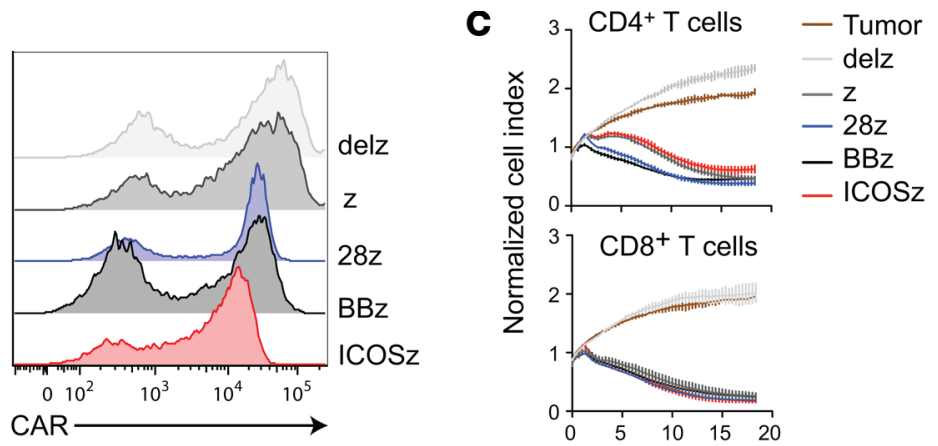

E
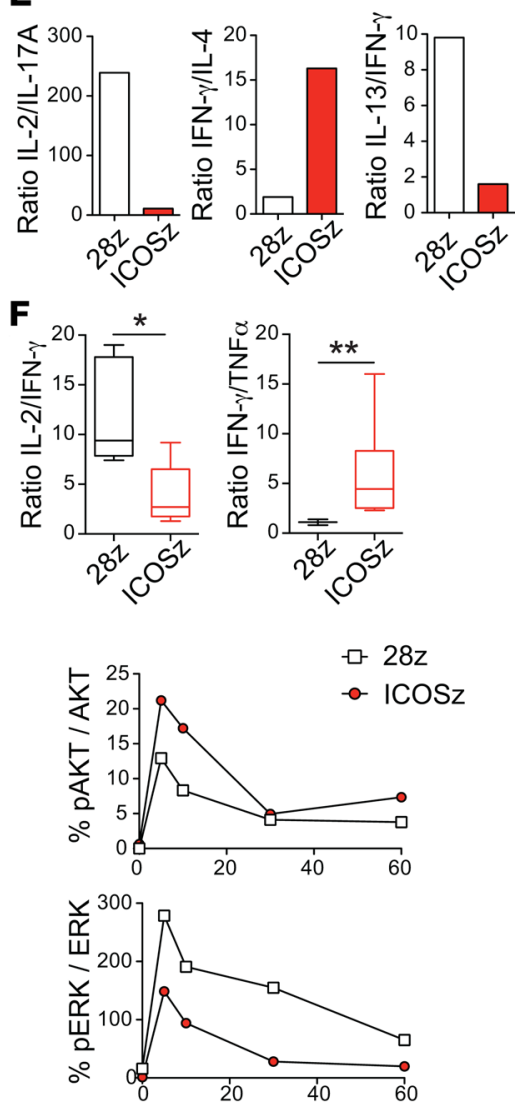

Minutes after antigen recognition

Figure 1. In vitro characterization of $\mathbf{C D 4}^{+}$and $\mathbf{C D 8}{ }^{+} \mathbf{T}$ cells redirected with SS1-CARs. (A) Schematic representation of chimeric receptors that contain the SS1 single chain fragment that binds to mesothelin and differ in the transmembrane and intracellular domains. (B) Surface expression of the SS1-CARs on human CD4+ $T$ cells at the end of the primary expansion. Representative of 3 donors. (C) A real-time, impedance-based cytotoxicity assay (xCelligence) was used to evaluate the lysis of non-small cell lung L55 tumor cells when treated with CAR T cells at 1:1 E:T ratio over a 20-hour period. Representative of 2 donors. (D) CD4+ CAR T cells were cocultured with pancreatic cancer cells (Capan-2) that express mesothelin. Supernatants were obtained 24 hours after coculture, and cytokine production was analyzed by Luminex. Representative of 3 different experiments performed under similar conditions. (E) Ratios of cytokine expression for $28 z$ and ICOSz were calculated using results from C. (F) CD4+ CAR T cells from different donors $(n=3-6)$ were cocultured with APC cells modified to express mesothelin. Supernatants were obtained 24 hours after coculture, and cytokine production was analyzed by ELISA. Ratios of cytokine expression for $28 \mathrm{z}$ and ICOSz are shown. Box plots show median (line) and 25 th to 75 th percentile (box). The end of the whiskers represents the minimum and the maximum of all of the data. ${ }^{*} P<0.05$ by t-test. (G) CD4+ CAR T cells were cocultured with Capan-2 or immobilized mesothelin. Supernatants were obtained 24 hours after coculture, and IL- 6 production was analyzed by Luminex. Representative of 3 donors $(\mathbf{H})$ CD4 ${ }^{+}$CAR T cells were stimulated with magnetic beads coated with recombinant mesothelin. Cell lysates were obtained at different time points $(5,10,30$ and $60 \mathrm{~min}$ ) and phosphorylation levels for AKT and ERK were analyzed by Western Blot and densitometry. Basal phosphorylation was evaluated without stimulation (minute 0). Representative of 3 donors. 
in $\mathrm{CD}^{+}$ICOSz cells (Figure 1F). Interestingly, CD4+ $\mathrm{BBz}$ CAR T cells released moderate levels of IL-6 that were consistently higher than those produced by CD4 $4^{+} 28 \mathrm{z}$ or CD $4^{+}$ICOSz cells (Figure $1 \mathrm{G}$ ). Those results were consistent when analyzed under different conditions and using different normal donors (Supplemental Figure 1). Importantly, similar trends for IL-2, IFN- $\gamma$, IL-13, and IL-5 expression were observed for $\mathrm{CD}^{+} \mathrm{CAR} \mathrm{T}$ cells (Supplemental Figure 1).

ICOS is a member of the CD28 family (23) and, similar to CD28, signals through the recruitment of PI3K and downstream activation of the MAP kinases JNK, p38, and ERK; however, ICOS signaling results in comparatively elevated phosphatidylinositol $(3,4,5)$-triphosphate (PIP3) levels and a concomitant increase in Akt signaling (24). To assess whether our CARs transduce intracellular signals in a manner similar to endogenous costimulatory molecules, AKT and ERK phosphorylation in response to antigen stimulation was analyzed by Western blot. Activation of $28 \mathrm{z}$ CAR T cell induced greater and more sustained pERK activity than activation of ICOSz CAR T cells (Figure $1 \mathrm{H}$ ). In contrast, signaling through ICOS increased AKT signaling relative to both CD3z and CD28z CAR T cells (Figure $1 \mathrm{H}$ and Supplemental Figure 1).

In summary, signaling though CD28- or ICOS-based CARs induced strikingly different phenotypes. Specifically, ICOSz CAR T cells displayed a Th1/Th17 phenotype with reduced levels of many Th1/Th2 cytokines, as well as increased pAKT signaling compared with $28 \mathrm{z}$ CAR T cells.

$C D 4^{+} T$ cells expressing an ICOS-based CAR displayed enhanced in vivo persistence and significantly increased the persistence of coinjected $C D 8^{+} T$ cells. Because robust in vivo expansion and long-term functional persistence of CAR T cells may be required to induce the destruction of large tumors, we next analyzed the in vivo persistence of CAR T cells in mice bearing tumors. To better understand the effect of distinct costimulatory domains in $\mathrm{T}$ cell survival, we cultured $\mathrm{CD} 4^{+}$and $\mathrm{CD}^{+} \mathrm{T}$ cells separately in vitro and redirected them with CARs containing different costimulatory signals (z, CD28, 4-1BB, ICOS). CD4 ${ }^{+}$and $\mathrm{CD}^{+} \mathrm{T}$ cell populations were then mixed to achieve a $1: 1 \mathrm{CD} 4^{+} / \mathrm{CD} 8^{+}$cell ratio and were injected into immunodeficient NOD/scid/IL $2 r \gamma^{-/-}$(NSG) mice bearing s.c. L55 non-small cell lung tumors. This experiment was designed to determine whether specific combinations of ICDs in each cell subset would enhance CD $4^{+}$and $\mathrm{CD}^{+}$CAR $\mathrm{T}$ cell persistence. Blood was obtained 22 days following the first $\mathrm{T}$ cell injection, and CD4 ${ }^{+}$ and $\mathrm{CD}^{+} \mathrm{T}$ cells were quantified. CD $4^{+} \mathrm{ICOSz} \mathrm{CAR} \mathrm{T}$ cells exhibited enhanced persistence compared with first-generation $\mathrm{CD}^{+}{ }^{+} \mathrm{z}$ CAR T cells, whereas $\mathrm{CD} 4^{+} 28 \mathrm{z}$ or BBz CAR T cells displayed impaired persistence (Figure 2A). Of note, enhanced persistence of $\mathrm{CD} 4^{+} \mathrm{ICOSz} C A R$ T cells was observed irrespective of which SS1-CAR was used to redirect the $\mathrm{CD} 8^{+} \mathrm{CAR} \mathrm{T}$ cells. In contrast, persistence of $\mathrm{CD} 8^{+} \mathrm{CAR} \mathrm{T}$ cells was highly dependent on the CAR used to redirect $\mathrm{CD} 4^{+} \mathrm{T}$ cells; specifically, the presence of $\mathrm{CD} 4^{+}$ ICOSz CAR T cells significantly increased the persistence of $\mathrm{CD}^{+} 28 \mathrm{z}$ or BBz CAR T cells (Figure 2B). The treatment group that exhibited the greatest $\mathrm{T}$ cell persistence was a combination of CD4 ${ }^{+} \mathrm{ICOSz}$ and $\mathrm{CD} 8^{+} \mathrm{BBz}$ CAR $\mathrm{T}$ cells, indicating that $\mathrm{CD} 4^{+}$and $\mathrm{CD} 8^{+} \mathrm{CAR} \mathrm{T}$ cells may require distinct costimulatory signals for optimal persistence.

To confirm these results using an independent $s c F v$, we repeated this experiment with $\mathrm{T}$ cells expressing the MOv19-CAR, which contains an scFv specific for folate receptor- $\alpha$. For this experiment, all $\mathrm{CD}^{+}$cells were redirected with the MOv19-BBz CAR, whereas CD4 ${ }^{+} \mathrm{T}$ cells were engineered to express MOv19-z, -ICOSz, -28z, or -BBz CARs. Two doses of CAR T cells were injected into NSG mice bearing large xenograft tumors derived from human SKOV3 ovarian cancer cells. Consistent with our SS1-CAR results, CD4+ ICOSz-MOv19 CAR T cells showed enhanced persistence, compared with corresponding $\mathrm{CD}^{+} \mathrm{T}$ cells expressing 28z- or BBz-MOv19 CARs (Figure 2C), and correlated with enhanced persistence of accompanying CD8 ${ }^{+}$BBz-MOv19 CAR T cells. All mice treated with CAR T cells showed robust antitumor responses (Figure 2D).

A third-generation CAR combining ICOS and 4-1BB signaling domains showed enhanced antitumor effect with increased in vivo persistence. The above data suggest that the ICOS ICD is critical for optimal persistence of $\mathrm{CD}^{+}$CAR T cells, whereas the 4-1BB ICD enhances CD8 ${ }^{+}$CAR T cell persistence when supported by $\mathrm{CD}^{+}$ICOSz CAR T cells. Next, we investigated whether combining these 2 costimulatory signals in a single, third-generation ICOSBBz CAR (Figure 1A) could enhance the function of adoptively transferred $\mathrm{T}$ cells. Both $\mathrm{CD}^{+}$and $\mathrm{CD}^{+}$human $\mathrm{T}$ cells expressed the ICOSBBz CAR, although overall surface expression was lower compared with second-generation CARs, consistent with previous reports of third-generation CARs. When cocultured with mesothelin-expressing tumor cell lines, $\mathrm{CD}^{+}{ }^{+}$ICOSBBz CAR T cells secreted levels of cytokines that were intermediate between 


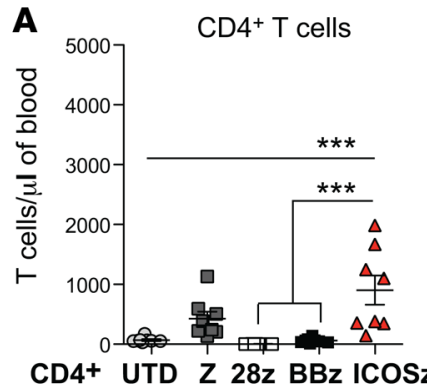

CD4+ UTD Z $28 z$ BBz ICOSz

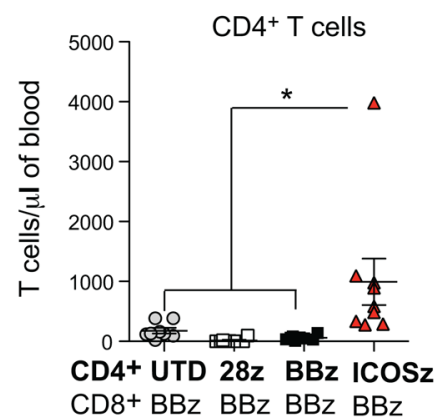

$\mathrm{CD} 8+\mathrm{BBz} \mathrm{BBz} \mathrm{BBz} \mathrm{BBz}$

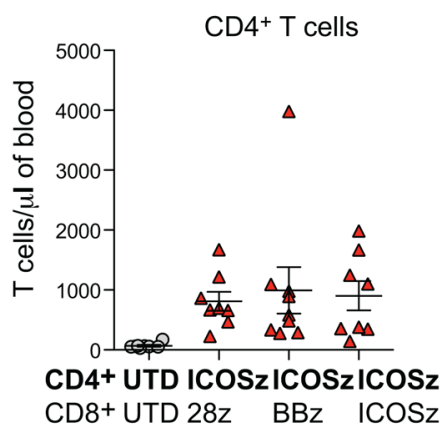

B

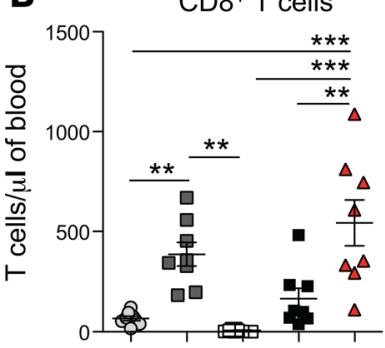

CD4+ UTD Z 28z BBz ICOSz CD8+ UTD Z 28z BBzICOSz
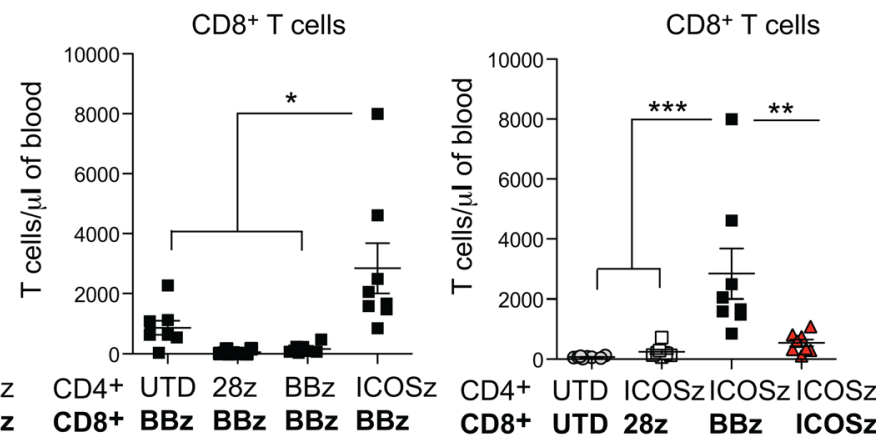

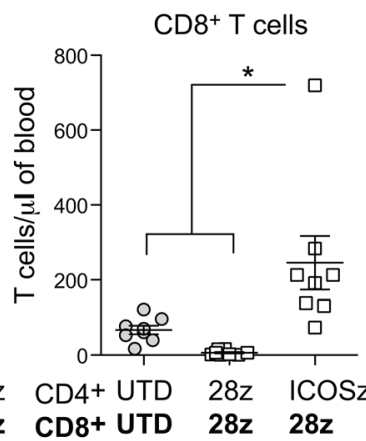

C

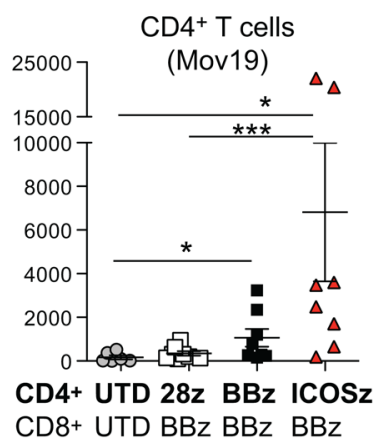

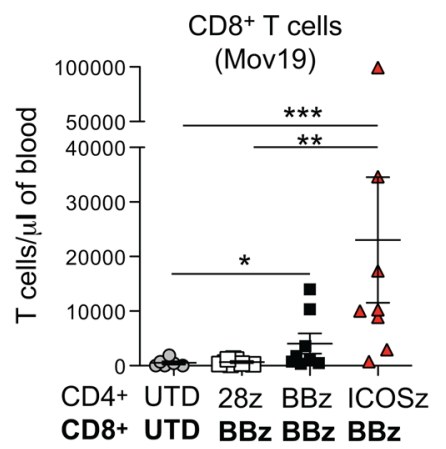

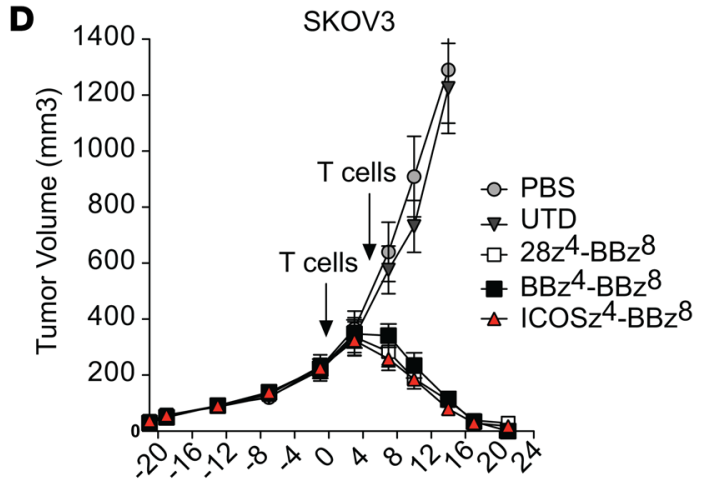

Days after $T$ cell injection

Figure 2. Helper effect of CD4+ CAR T cells expressing an ICOS-based CAR significantly increased the persistence of coinjected CD8 ${ }^{+}$CAR T cells expressing 4-1BB- or CD28-based CARs. (A and B) NSC mice bearing s.c. L55 tumor cells were treated 30 days after tumor implantation with 2 doses of $\mathrm{CD}^{+}$and $\mathrm{CD} 8^{+} T$ cells redirected with SS1-CARs. The concentration of $(\mathbf{A}) \mathrm{CD} 4^{+} \mathrm{T}$ cells and $(\mathbf{B}) \mathrm{CD} 8^{+} \mathrm{T}$ cells was determined in the blood of treated animals 22 days after $T$ cell injection. Error bars represent $\pm \operatorname{SEM}(n=7-10)$. ${ }^{*} P<0.05$; ${ }^{* *} P<0.01$, and ${ }^{* * *} P<0.001$ by 1 -way ANOVA with Tukey post hoc test. (C and D) NSG mice bearing s.c. ovarian tumors (SKOV3) were treated 30 days after tumor implantation with 2 doses of CD4 $4^{+}$and $C D 8^{+}$T cells redirected with anti-folate receptor $\alpha$ Mov19-CARs. CD8 ${ }^{+} T$ cells were modified to express BBz, and CD4+ ${ }^{+}$cells were left untransduced or redirected with Z, 28z, $\mathrm{BBz}$, or ICOSz signaling domains. (C) The concentration of CD4+ and CD8+ $\mathrm{T}$ cells were determined in the blood of treated animals 27 days after $\mathrm{T}$ cell injection. Error bars represent \pm SEM $(n=6-8)$. T cell count data was transformed to reduce variance, and significance was analyzed by 1 -way ANOVA with Tukey post hoc test. (D) Tumor volume was analyzed at indicated time points; legend indicates the signaling domain in the CD4+ and CD8 ${ }^{+} \mathrm{T}$ cells. Results are expressed as a mean tumor volume $( \pm$ SEM) with $n=6-8$ mice per group.

those observed for $\mathrm{CD}^{+}{ }^{+} \mathrm{ICOSz}$ and $\mathrm{CD} 4^{+} \mathrm{BBz}$ CAR T cells, indicating that the effects of combining these ICDs in 1 molecule was not simply additive (Supplemental Figure 2). Overall, no major differences were observed among groups.

We next investigated the antitumor efficacy of ICOSBBz compared with the second-generation CARs described in Figure 1. As a positive control, we included a treatment group containing combined $\mathrm{CD}^{+}$ ICOSz and $\mathrm{CD}^{+} \mathrm{BBz}$ CAR T cells $\left(\mathrm{ICOSz}^{4}-\mathrm{BBz}^{8}\right)$. After establishing s.c. Capan- 2 cell-derived pancreatic xenograft tumors in NSG mice for 15 days, mice received 2 doses of redirected CAR T cells. Bulk T cells (containing both $\mathrm{CD}^{+}$and $\mathrm{CD} 8^{+}$cells) redirected with either ICOSz or BBz CARs slowed tumor growth but did not result in major tumor regression (Figure 3, A and B). The combination of $\mathrm{ICOSz}^{4}-\mathrm{BBz}^{8} \mathrm{CAR}$ 
A

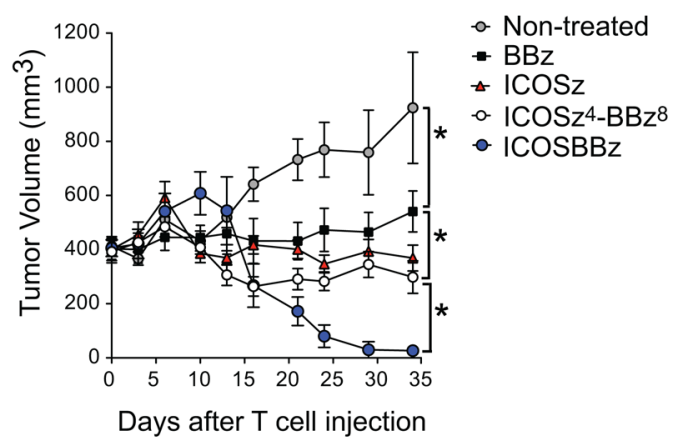

B

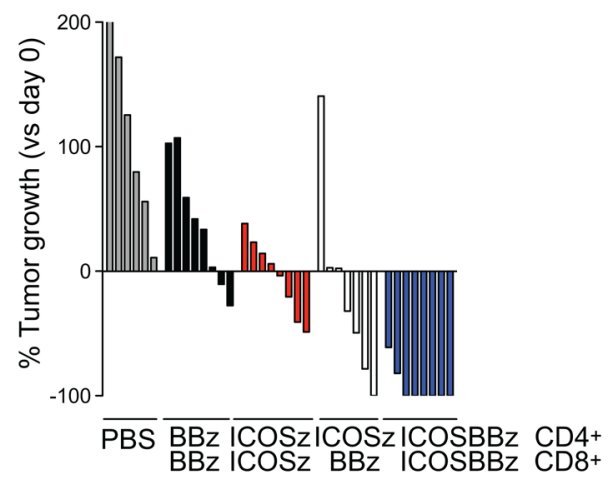

C

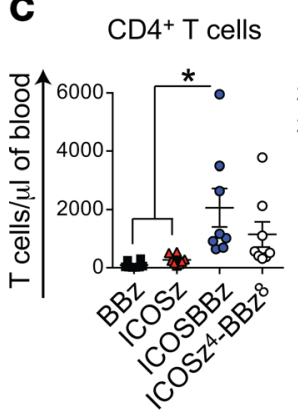

CD8 $+T$ cells

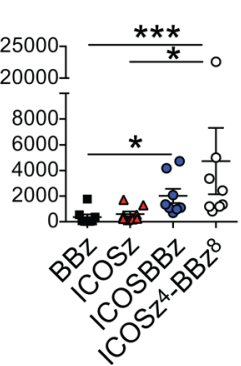

D

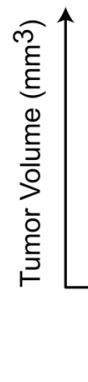

ICOSz4-BBz 8

E

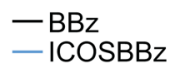

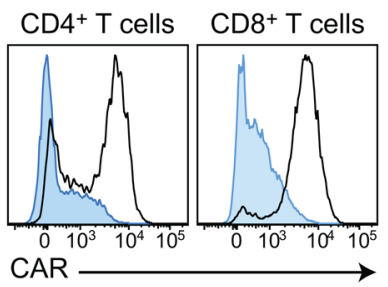

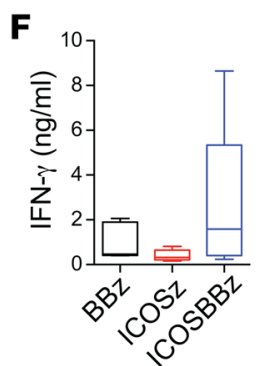
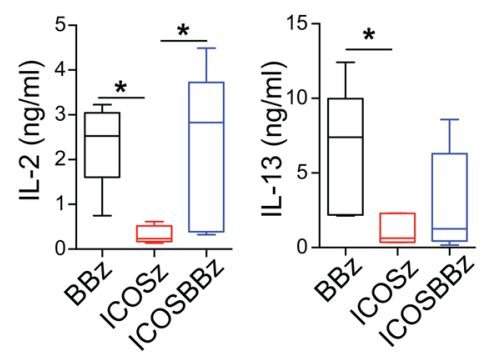

$\mathrm{CD}^{+} \mathrm{T}$ cells

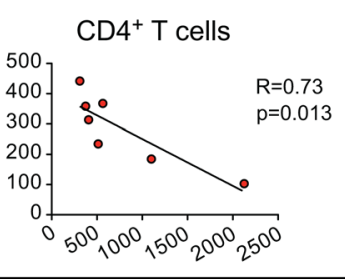

$\mathrm{T}$ cells $/ \mu \mathrm{l}$ of blood
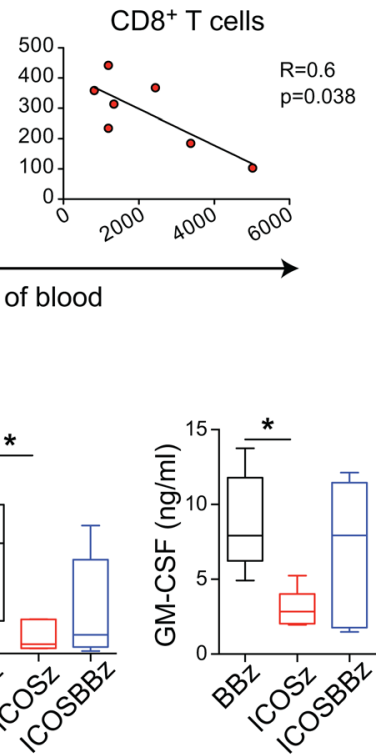

Figure 3. Third-generation CARs containing ICOS and 4-1BB intracellular domains mediate enhanced antitumor effects and increased T cell persistence. (A) NSG mice bearing s.c. pancreatic tumors (Capan-2) were treated 15 days after tumor implantation with 2 doses of T cells redirected with SS1-CARs. A

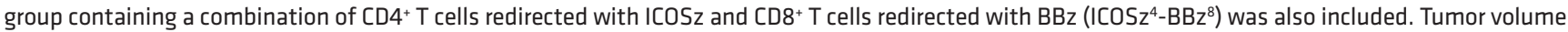
was analyzed at indicated time points. Results are expressed as a mean tumor volume ( \pm SEM) with $n=6-8$ mice per group. ${ }^{*} P<0.05$ by 2 -way ANOVA with Tukey's multiple comparison test. (B) Waterfall plots of the change in tumor volume on day 34 versus baseline for individual animals. (C) The concentration of $\mathrm{CD}^{+}$and $\mathrm{CD}^{+} \mathrm{T}$ cells were determined in the blood of treated animals 21 days after $\mathrm{T}$ cell injection. Error bars represent $\pm \mathrm{SEM}$. ${ }^{*} P<0.05$ by 1 -way ANOVA with Tukey post hoc test. ${ }^{* *} P<0.001$. For $C D 8^{+} T$ cell counts, data was transformed to reduce variance, and significance was analyzed by 1 -way ANOVA with Tukey post hoc test. (D) Correlation of numbers of $\mathrm{CD} 4^{+}$and $C D 8^{+} T$ cells per microliter of blood was plotted versus the tumor volume on day 21 for the animals treated with ICOSz $z^{4}-B B z^{8}$. (E and F) NSG mice were sacrificed on day 34 after treatment, and human T cells were isolated from mouse spleens. (E) CAR expression was analyzed by flow cytometry. Representative of 3-5 animals. (F) CAR T cells recovered from spleen were cocultured with K562 that express mesothelin, and cytokine release was analyzed by ELISA 24 hours after coculture. Box plots show median (line) and 25th to 75 th percentile (box). The end of the whiskers represents the minimum and the maximum of all of the data. ${ }^{*} P<0.05$ by 1 -way ANOVA with Tukey post hoc test.

T cells caused initial rapid tumor regression and prevented further tumor growth, although tumors were not eradicated. Mice treated with mixed ICOSz $-\mathrm{BBz}^{8} \mathrm{CAR} \mathrm{T}$ cells displayed enhanced persistence of both $\mathrm{CD}^{+}$and $\mathrm{CD}^{+}$components, relative to mice treated with bulk ICOSz or BBz CAR T (Figure 3C), and correlated directly with antitumor activity (Figure 3D). Interestingly, mice injected with bulk ICOSBBz CAR T cells displayed an initial increase in tumor size, after which tumors regressed dramatically such that tumor eradication occurred in $75 \%$ of the mice 4 weeks after treatment (Figure $3, \mathrm{~A}$ and $\mathrm{B}$ ). The robust antitumor effect of ICOSBBz CAR T cells was accompanied by increased numbers of both $\mathrm{CD}^{+}$and $\mathrm{CD}^{+} \mathrm{T}$ cells when compared with second-generation CARs (Figure $3 \mathrm{C}$ ).

We next characterized the phenotype and function of persisting ICOSBBz CAR T cells obtained from the spleens of treated animals at the end of the study (Day 35). ICOSBBz CAR T splenocytes showed lower frequencies and surface-expression levels of CAR protein (Figure 3E); however, these cells produced 
similar levels of cytokines as BBz CAR T splenocytes following coculture with mesothelin-expressing antigen presenting cells (APCs) (Figure 3F).

Surface levels of CAR expression influences antitumor effects in vivo. Next, we sought to investigate the mechanism behind the enhanced antitumor effect of ICOSBBz CAR T cells. Previous studies suggested that elevated CAR expression can induce antigen-independent tonic signaling, which can promote $\mathrm{T}$ cell exhaustion and limit the potency of CAR T cells $(25,26)$. Therefore, we hypothesized that the lower mean CAR expression in ICOSBBz T cells could prevent T cell exhaustion and enhance antitumor effects. To test this hypothesis, we attempted to equilibrate T cell surface expression of ICOSBBz and BBz CARs. Specifically, we first transduced $\mathrm{T}$ cells with the corresponding lentiviral vector (LV) at different multiplicities of infection (MOI). Increasing the MOI elevated the percentage of T cells expressing the ICOSBBz to greater than $80 \%$; however, cell-surface CAR expression remained significantly lower than that of BBz CAR T cells at every MOI tested (Figure 4A). In order to assess whether the elevated CAR expression observed in BBz CAR T cells resulted in increased tonic signaling, we measured the mean cell volumes following primary expansion as a measurement of metabolic status and cell cycle. Mean T cell volume was higher in BBz CAR T cells compared with all other groups, consistent with 4-1BB signaling in the absence of cognate antigen (Figure 4B). Increasing the viral MOI used to transduce T cells also increased $\mathrm{T}$ cell volume in all groups, confirming previous reports that elevated cell-surface CAR expression contributes to tonic signaling.

Next, we attempted to equilibrate $\mathrm{T}$ cell surface expression of ICOSBBz and BBz CARs using a less active promoter to drive $\mathrm{BBz}$ expression. Specifically, we replaced the EF-1 $\alpha$ promoter, which was used in all other LV vectors in our study, with a truncated promoter from the Phosphoglycerate kinase (Pgk) gene, designated pGK300 (26). T cells transduced with the modified pGK300-BBz LV displayed cell surface CAR expression levels similar to those observed for EF-1 $\alpha-$ ICOSBBz CAR T cells (Figure 4, C and D). To analyze the effect of CAR expression levels on tonic signaling, we monitored the mean $\mathrm{T}$ cell volume and $\mathrm{T}$ cell phenotype after activation with anti-CD3 and anti-CD28 beads, in the absence of cognate antigen. After T cell activation, ICOSBBz cell volume increased to nearly $600 \mathrm{fl}$ by day 5 of culture and returned to a resting cell volume of less than $250 \mathrm{fl}$, similar to untransduced (UTD) T cells, by days 10-11 (Figure 4E). By day 14 following activation, ICOSBBz T cells showed a T cell phenotype similar to that of UTD T cells, with no signals of $\mathrm{T}$ cell activation, exhaustion, or differentiation (Figure $4 \mathrm{~F}$ ). In contrast, when BBz expression was driven by the EF-1 $\alpha$ promoter, T cells failed to return to a resting cell volume within 12 days of culture, and by day 14, EF-1 $\alpha$-BBz cells still expressed high levels of IL-2 receptor $\alpha$ CD25. EF-1 $\alpha$-BBz cells showed reduced levels of CD28 and CD27 - 2 markers that typically decrease throughout differentiation - and higher frequencies of the inhibitory receptors PD1, TIM-3, and CD200, typically expressed on chronically stimulated T cells. As previously suggested, lowering baseline CAR expression reduced tonic costimulation, as BBz expression from the less-active pGK300 promoter normalized the T cell phenotype (Figure 4F).

Next, we sought to analyze whether reducing the levels of $\mathrm{BBz}$ expression could result in an enhanced antitumor effect. Surprisingly, when administered into animals bearing xenograft pancreatic tumors, pGK300$\mathrm{BBz} \mathrm{T}$ cells were unable to induce any antitumor effect relative to UTD T cell controls (Figure 4G), suggesting that lower CAR expression is not generally beneficial. In contrast, although ICOSBBz CAR T cells expressed significantly less cell-surface CAR protein than EF-1 $\alpha$-BBz CAR T cells, they were significantly more proficient at controlling tumor growth (Figure $4 \mathrm{G}$ ). These results demonstrate that the superior antitumor efficacy of the ICOSBBz CAR is not simply a consequence of its lower surface expression and its reduced tonic signaling.

ICOS proximity to the cell membrane in a third-generation CAR is necessary for improved survival. We hypothesized that, if combining ICOS and 4-1BB signaling enhances antitumor effect, a third-generation CAR containing both ICOS and 4-1BB in reversed orientation (BBICOSz), in which the 4-1BB domain is positioned proximal to (and the ICOS domain distal to) the plasma membrane should also show superior antitumor effect when compared with second-generation CARs. To test this, we generated BBICOSz CAR T cells (Figure 1A) and compared their in vitro phenotype and function to BBz, ICOSz, and ICOSBBz CAR T cells. All second- and third-generation CARs demonstrated equivalent kinetics of target cell lysis in vitro (data not shown). Incorporation of the 4-1BB intracellular domain in third-generation CARs slightly increased IL-2 secretion and $\mathrm{T}$ cell proliferation when compared with ICOSz T cells in the 3 normal donors tested (Figure 5, A and B). Interestingly, cytokine secretion patterns were most similar between BBICOSz and BBz cells, as well as between ICOSBBz and ICOSz cells. For example, BBICOSz and BBz cells exhibited significantly higher IL-13 and IL-6 secretion than ICOSz and ICOSBBz cells, whereas the reverse pattern was observed for IL-10 and IL-17 (Figure 5A). These data suggest that the membrane-proximal ICD has an important effect on cytokine responses. 
A

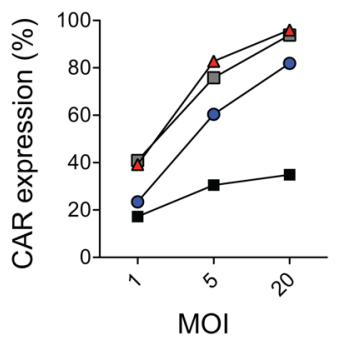

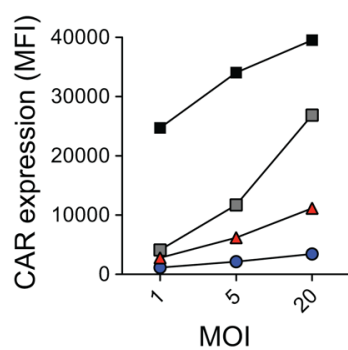

B

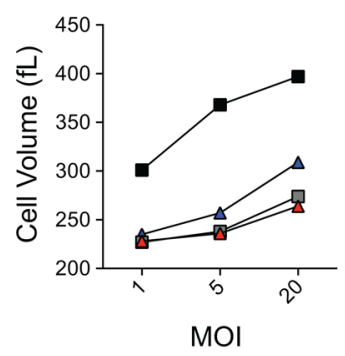

-ㅁ $Z$

$\rightarrow \mathrm{BBz}$

$\triangle 1 \mathrm{COSz}$

- $1 \mathrm{ICOSBBz}$

c

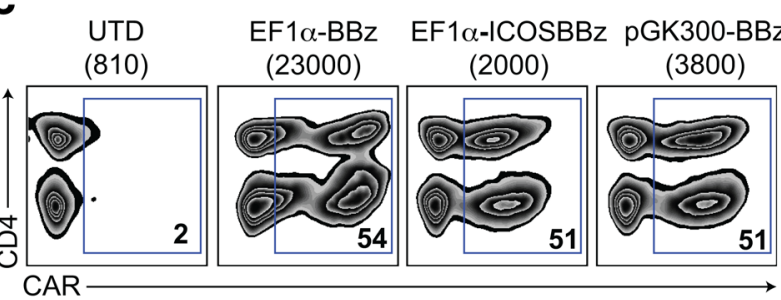

E

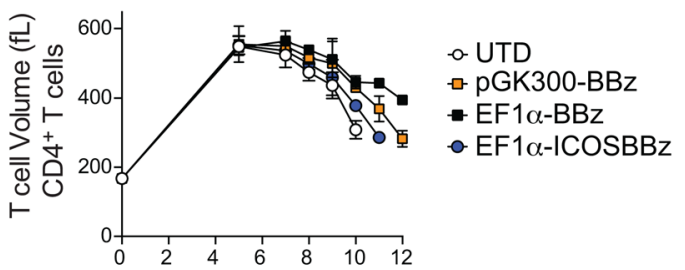

Days after $\alpha C D 3 / C D 28$ stimulation

G

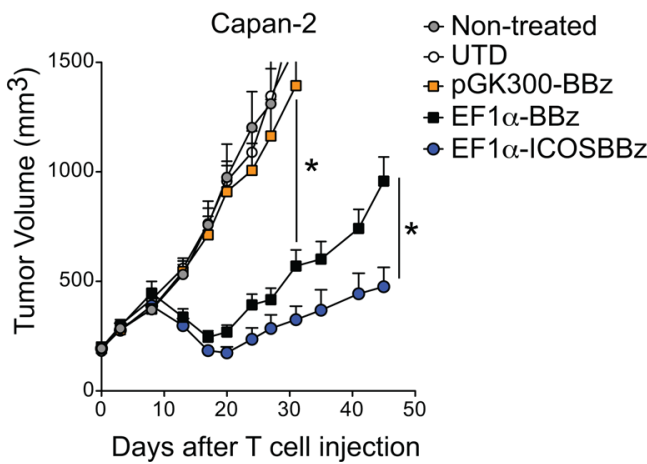

$\mathbf{F}$

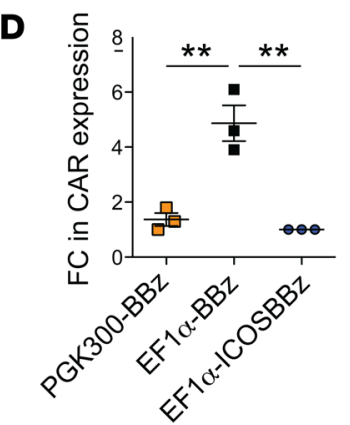

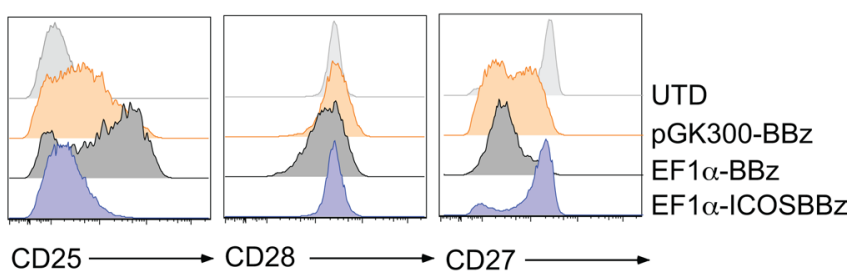
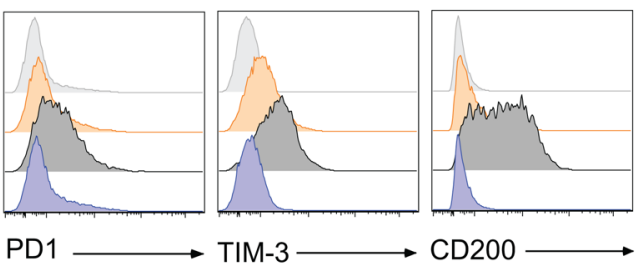

UTD pGK300-BBz $\mathrm{EF} 1 \alpha-\mathrm{BBz}$ EF $1 \alpha-I C O S B B z$<smiles>CCCCCCCC</smiles>

Figure 4. Levels of surface CAR expression influence antitumor effects. (A and B) T cells were transduced with indicated MOI with lentiviral vectors encoding CAR proteins. CAR expression (A) and mean cell volume (B) was analyzed on day 13 after T cell transduction by flow cytometry. (C) Surface expression of the CAR proteins on human T cells at the time of functional evaluation. Transduction efficiencies are indicated with MFI of the transduced populations in parentheses. (D) Fold-change CAR expression (MFI) in pGK300-BBz and EF-1 $\alpha$-BBz relative to EF-1 $\alpha-I C O S B B z$ was analyzed in 3 donors. ${ }^{*} P<0.01$ by 1-way ANOVA with Tukey post hoc test. (E) T cell volume during ex vivo expansion in the absence of cognate antigen. Results are expressed as the mean T cell volume ( $\pm \mathrm{SD}$ ) with $n=2$ donors. (F) Representative histograms showing the expression of activation, differentiation, and exhaustion markers in CAR T cell 14 days after stimulation with anti-CD3/CD28 beads. Representative of 2 donors. (C) NSG mice bearing s.c. Capan-2 pancreatic tumors were treated 20 days after tumor implantation with 2 doses of UTD or CAR T cells with the indicated promoter and signaling domain. Tumor volume was analyzed at indicated time points. Results are expressed as the mean tumor volume $( \pm$ SEM) with $n=7-10$ mice per group. Statistical significance: ${ }^{*} P<0.05$ by 2 -way ANOVA with Tukey's multiple comparison test.

To determine the antitumor effect of BBICOSz, NSG mice bearing pancreatic tumors were treated with redirected $\mathrm{T}$ cells $\left(50 \% \mathrm{CAR}, 1: 1 \mathrm{CD}^{+} / \mathrm{CD}^{+}\right)$, and overall survival was analyzed (Figure $\left.5 \mathrm{C}\right)$. Injection of $\mathrm{BBz}$ or BBICOSz cells effectively doubled the median survival of NSG mice bearing s.c. xenograft pancreatic tumors, compared with UTD controls. Remarkably, $100 \%$ of NSG mice receiving ICOSz or ICOSBBz CAR $\mathrm{T}$ cells were alive by day 56 , compared with only $40 \%$ survival in the BBz and BBICOSz treatment groups. 
A
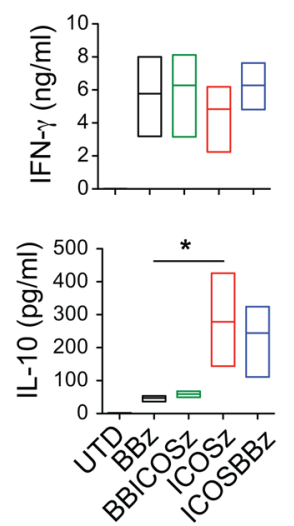

B

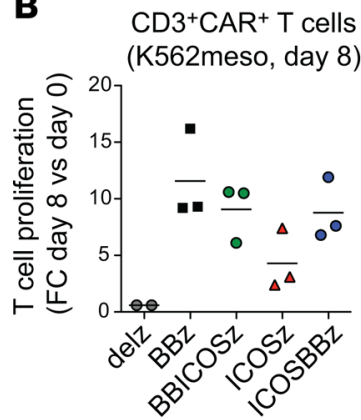

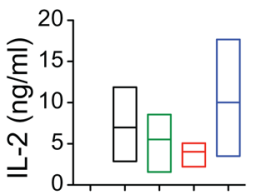

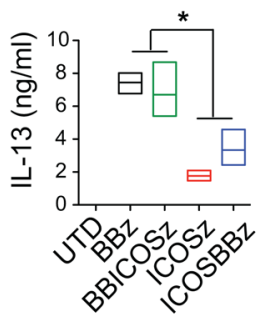

C
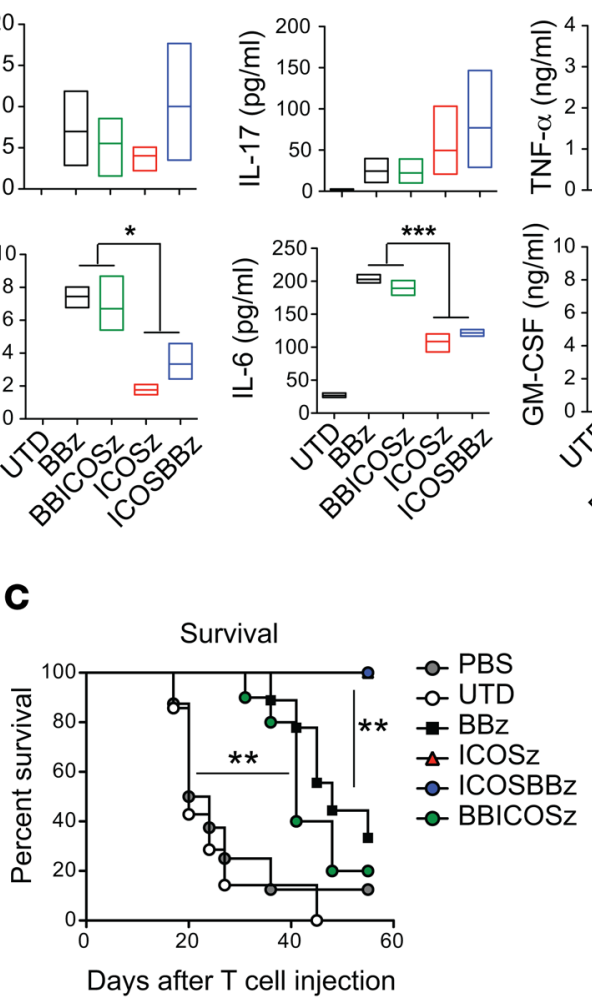
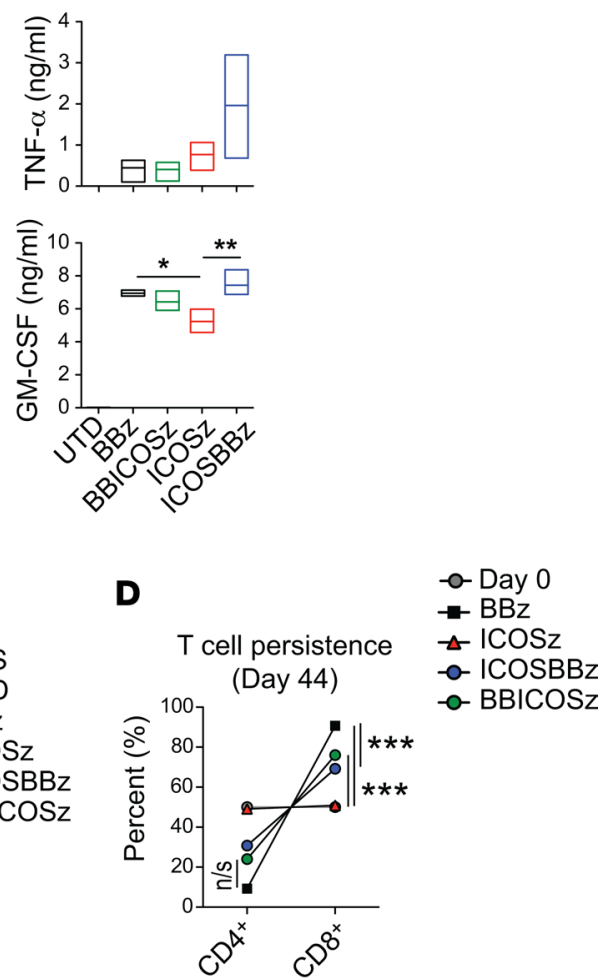

Figure 5. ICOSBBz enhanced in vivo functions are only observed when ICOS is proximal to the cell membrane. (A and B) CAR T cells obtained from 3 different healthy donors were cocultured with APC cells transduced with mesothelin (K562meso). (A) Supernatants were obtained 24 hours later, and cytokine production was analyzed by Luminex. Floating bars show mean (line) and the minimum and maximum values (box). ${ }^{*} P<0.05$, ${ }^{* *} P<0.01$, and ${ }^{* * *} P$ $<0.001$ by 1 -way ANOVA with Tukey post hoc test. (B) Proliferation was assessed by determining the total number of live CAR T cells within the coculture flow cytometry using counting beads 8 days after coculture. Total cell count at the end of the experiment was normalized to the total cell count at day 0. (C and D) NSC mice bearing s.c. pancreatic tumors (Capan-2) were treated 20 days after tumor implantation with 2 doses of UTD or CART cells ( $n=7-10$ mice per group). (C) Kaplan-Meier survival curves are plotted. The predetermined end point was established when tumor volume exceeded 1,000 mm ${ }^{3}$. ${ }^{*} P<0.01$ by a log rank (Mantel-Cox) test. (D) The ratio of CD4+ and CD8 ${ }^{+}$cells within the human CD45 ${ }^{+} \mathrm{T}$ cell population in the peripheral blood of treated animals was analyzed on day 44 by flow cytometry ( $n=7-10$ mice per group). ${ }^{* *} P<0.001$ by 1 -way ANOVA with Tukey post hoc test.

Analyses conducted 44 days after injection revealed that more than $90 \%$ of surviving $\mathrm{T}$ cells in animals treated with BBz T cells were $\mathrm{CD}^{+}$, whereas $50 \%$ of the persisting ICOSz T cells were CD4 $4^{+}$(Figure $5 \mathrm{D}$ ). These data suggest that 4-1BB signaling is critical for the survival of CD8 ${ }^{+} \mathrm{CAR} T$ cells, and ICOS signaling is critical for the survival of $\mathrm{CD}^{+} \mathrm{CAR}$ T cells. Animals treated with third-generation CARs containing both ICOS and 4-1BB showed an intermediate pattern, composed of $70 \%-80 \%$ of $\mathrm{CD}^{+} \mathrm{CAR} \mathrm{T}$ cells (Figure $5 \mathrm{D}$ ). Collectively, these results indicate that ICOS proximity to the cell membrane is key to enhancing antitumor effects, whereas incorporation of $4-1 \mathrm{BB}$ in $\mathrm{CD}^{+} \mathrm{T}$ cells is required to enhance proliferation and elevate the $\mathrm{CD} 8^{+} /$ $\mathrm{CD} 4^{+}$ratio in vivo.

ICOS TM domain regulates antitumor effect and increases persistence. Although the identity of the membrane-proximal ICD has a strong influence on CAR T cell function and persistence, it remained possible that the different TM domains used to construct ICOSBBz and BBICOSz CARs contributed to their phenotype. To test this, we created an additional third-generation CD8-ICOSBBz CAR in which the ICOS TM domain in the ICOSBBz CAR was replaced with the CD $8 \alpha$ TM used to produce the BBICOSz CAR (Figure 1A). Surface expression of the CD8-ICOSBBz CAR was very similar to that of ICOSBBz CAR T cells in all donors tested (Figure 6, A and B), suggesting that the TM domain does not have a key role on cell-surface CAR expression.

To investigate whether the TM domain could be responsible for tonic CAR signaling, we analyzed the mean cell volume and $\mathrm{T}$ cell phenotype during the primary expansion, in the absence of cognate antigen. All CAR constructs containing the ICOS ICD proximal to the cell membrane showed growth curves and phenotypes similar to control cells (Figure 6, C and D). Replacement of the ICOS TM domain by the CD8 $\alpha$ TM did not have any effect in tonic signaling. By contrast, both BBz and BBICOSz CAR T cells showed increased mean cell volume during the primary expansion (Figure 6C). 
A

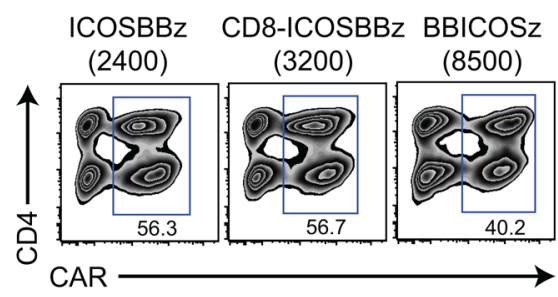

B

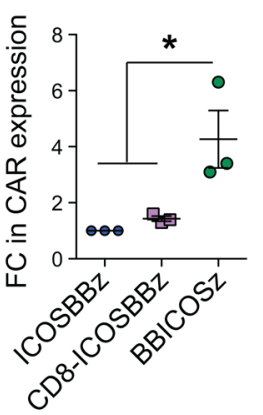

C

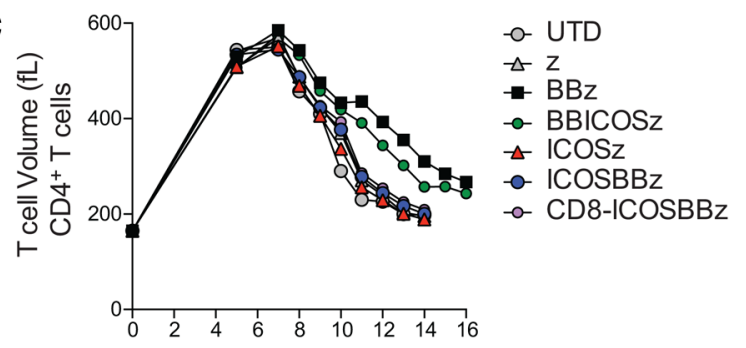

Days after $\alpha \mathrm{CD} 3 / \mathrm{CD} 28$ stimulation
D

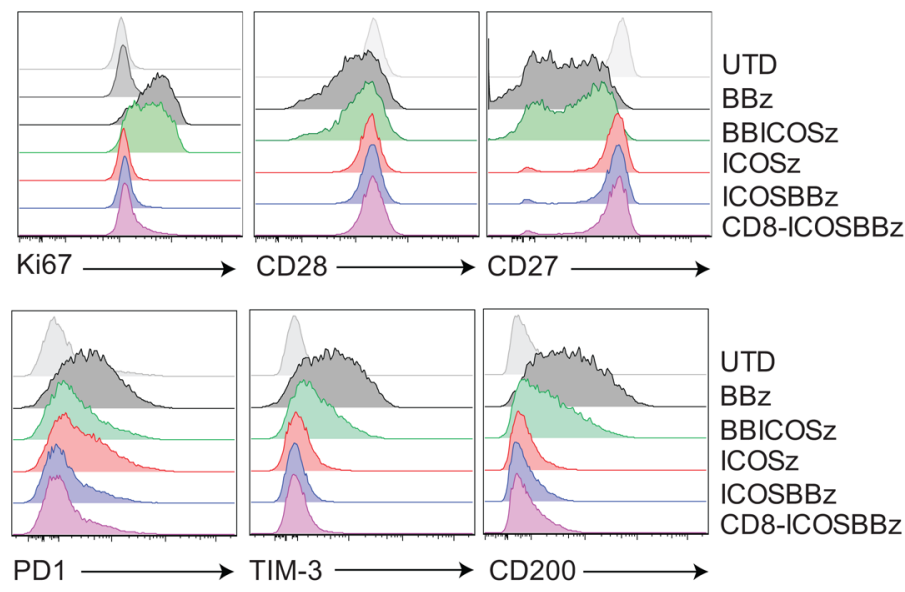

$\mathbf{F}$

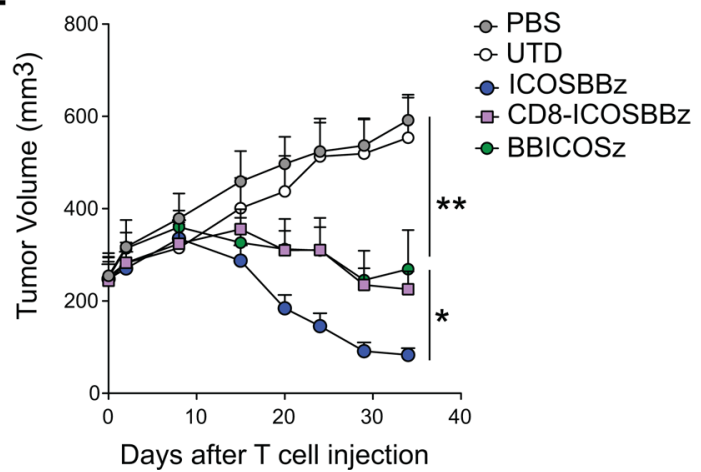

E
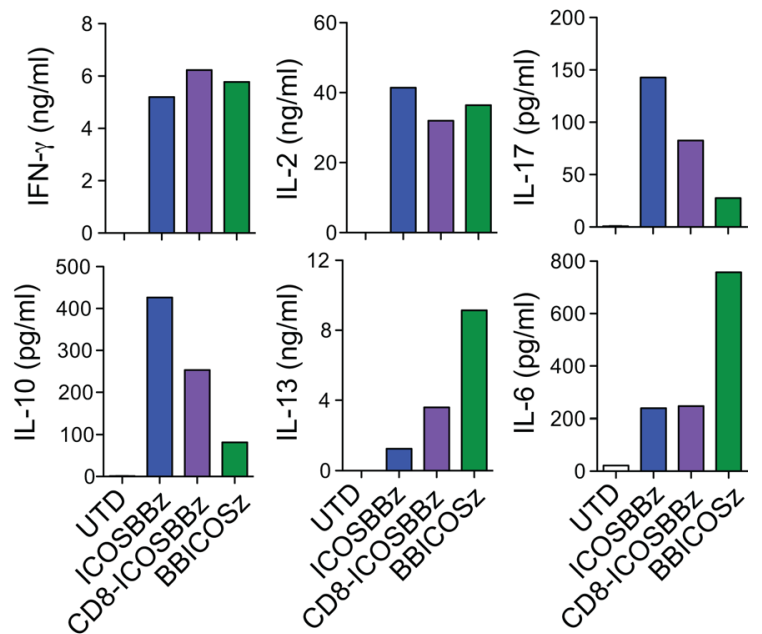

G
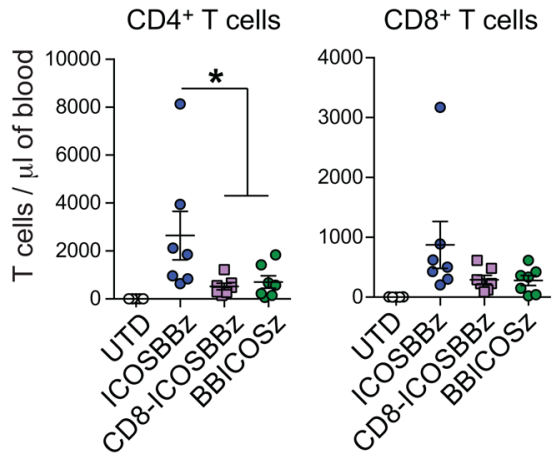

Figure 6. ICOS transmembrane domain is necessary for improved antitumor effect and increased persistence. (A) Surface expression of the CAR proteins on human T cells at the time of functional evaluation. ICOSBBz has an ICOS transmembrane region and TM CD8-ICOSBBz and BBICOSz have a CD8 $\alpha$ transmembrane domain. Transduction efficiencies are indicated with MFI of the transduced populations in parentheses. (B) Fold-change CAR expression (MFI) in CD8-ICOSBBz and BBICOSz relative to ICOSBBz was analyzed in 3 normal donors. ${ }^{*} P<0.05$ by 1 -way ANOVA with Tukey post hoc test. (C) T cell volume during ex vivo expansion in the absence of cognate antigen. Representative of 2-4 donors (D) Representative histograms showing the expression of activation, differentiation, and exhaustion markers in CAR T cells 13 days after stimulation with anti-CD3/CD28 beads. Representative of 2 donors. (E) CAR T cells were cocultured with APC cells transduced with mesothelin (K562meso). Supernatants were obtained 24 hours later, and cytokine production was analyzed by Luminex. (F and $\mathbf{G})$ NSG mice bearing s.c. Capan-2 pancreatic tumors were treated 36 days after tumor implantation with 2 doses of UTD or CAR T cells. (F) Tumor volume was analyzed at indicated time points. Results are expressed as a mean tumor volume $\left( \pm\right.$ SEM) with $n=5-7$ mice per group. ${ }^{*} P<0.05,{ }^{*} P$ $<0.01$ by 2-way repeated-measure ANOVA. (C) The concentration of CD4 $4^{+} T$ cells and $C D 8^{+} T$ cells was determined in the blood of treated animals 21 days after $T$ cell injection. Error bars represent \pm SEM $(n=5-7)$. To analyze differences among groups, $T$ cell count data was transformed to reduce variance, and significance was analyzed by 1 -way ANOVA with Tukey post hoc test; ${ }^{*} P<0.05$.

By day 13 after $\mathrm{T}$ cell activation with $\mathrm{CD} 3 / \mathrm{CD} 28$ beads, when most of the groups had rested down, $\mathrm{BBz}$ and BBICOSz groups showed signs of proliferation (Ki67 $\left.{ }^{\text {hi }}\right)$, differentiation $\left(\mathrm{CD} 28^{\mathrm{lo}}, \mathrm{CD} 27^{10}\right)$ and exhaustion (PD1 ${ }^{\text {hi }}, \mathrm{TIM}-3^{\mathrm{hi}}$, and CD200 ${ }^{\mathrm{hi}}$ ), suggesting that 4-1BB proximity to the cell membrane could account for the tonic signaling phenotype (Figure 6D). 
In vitro functional studies did not reveal any notable differences between the CD8-ICOSBBz and original ICOSBBz CAR T cells in terms cytotoxicity (data not shown) or cytokine release (Figure 6E) following antigen encounter. Notably, BBICOSz CAR T cells expressed higher levels of IL-13 and IL-6 when compared with TM CD8-ICOSBBz confirming that the membrane-proximal ICD has an important role in driving the cytokine profile of this third-generation CAR.

Despite the absence of the tonic signaling phenotype in the CD8-ICOSBBz CAR T cells, CD8-ICOSB$\mathrm{Bz}$ and BBICOSz cells displayed similar, modest antitumor effects in vivo (Figure 6F), whereas ICOSBBz cells caused $100 \%$ tumor regression by 35 days. Importantly, ICOSBBz CAR T cells showed an enhanced expansion and persistence in vivo (Figure 6G) when compared with CD8-ICOSBBz and BBICOSz cells. These data indicate that both the ICOS TM and ICD domains contribute to the robust antitumor effect and enhanced persistence in vivo observed for ICOSBBz CAR T cells.

In summary, our third-generation ICOSBBz CAR displayed enhanced persistence of $\mathrm{CD} 4^{+}$and $\mathrm{CD} 8^{+}$ CAR T cell subsets and superior antitumor activity and survival in solid tumor models compared with other CARs tested, notably the BBz CAR - which, when targeting CD19, has achieved long-term remissions in patients with $\mathrm{B}$ cell malignancies.

\section{Discussion}

The in vivo expansion and persistence of CAR T cells correlates with clinical response to B cell malignancies; however, CAR T cells have not persisted well in patients with solid tumors or demonstrated robust efficacy in trials reported to date. Herein, we investigated whether CAR T cell persistence and efficacy could be improved by redirecting $\mathrm{CD}^{+}$and $\mathrm{CD}^{+}$cells with optimized costimulatory domains. CAR $\mathrm{T}$ cell functionality is commonly studied in bulk $\mathrm{CD} 4^{+}$and $\mathrm{CD} 8^{+} \mathrm{T}$ cells. However, there is compelling evidence that costimulatory signals may have differential effects on $\mathrm{CD}^{+}$and $\mathrm{CD}^{+}$cells (27). While ICOS costimulation plays an essential role in the function of $\mathrm{CD}^{+} \mathrm{T}$ cells, 4-1BB signaling is known to preferentially enhance the survival of $\mathrm{CD}^{+} \mathrm{T}$ cells $(28,29)$. Here, we find that CAR $\mathrm{T}$ cells can have a "helper effect", in that the redirection of $\mathrm{CD} 4^{+} \mathrm{T}$ cells with an ICOS-based CAR enhances CD8 ${ }^{+} \mathrm{CAR}$ $\mathrm{T}$ cell persistence and killing in multiple in vivo solid tumor models. A third-generation CAR composed of ICOS and 4-1BB ICD was shown to have the most potent antitumor efficacy in preclinical solid tumor models, which is notable because it is more potent than the second-generation CAR that we previously developed that contains only $4-1 \mathrm{BB}$ and $\mathrm{CD} 3 \zeta \mathrm{ICD}(30)$ and has potent effects in patients with refractory leukemia (4-6). The underlying mechanisms by which ICOS and 4-1BB signaling differentially promote proliferation of $\mathrm{CD}^{+}$and $\mathrm{CD} 8^{+} \mathrm{CAR} \mathrm{T}$ cells may be related to distinct patterns of cytokine secretion and other effects that are currently under investigation.

Our results show that redirection of $\mathrm{CD} 4^{+} \mathrm{T}$ cells with a second-generation CAR containing the ICOS ICD drives T cells toward a Th1/T17 phenotype, with increased production of IL-17A, reduced IL-2 secretion, and enhanced in vivo survival when compared with second-generation CARs containing the widely used CD28 or 4-1BB ICD. We also demonstrate that the CD28-based CAR T cells selectively secrete TNF- $\alpha$, while 4-1BB-based CARs have preferential secretion of IL-6, which may be related to an emerging clinical spectrum of adverse effects with CD19 CARs, including cerebral edema and cytokine release syndrome (31).

These data extend our previous report demonstrating that Th17 cells redirected with an ICOS-based CAR had enhanced CD4 ${ }^{+} \mathrm{T}$ cell survival and were as effective as CD28-based CARs in killing tumor cells (21). These results also correlate with clinical observations in ICOS-deficient patients, whose $\mathrm{CD}^{+} \mathrm{T}$ cells exhibit normal IL-2 production but reduced secretion of the cytokines IL-4, IFN- $\gamma$, IL-10, and IL-17 upon in vitro stimulation, as well as a decreased percentage of $\mathrm{CD} 4^{+}$central and effector memory $\mathrm{T}$ cells (32). Interestingly, the percentage of ICOS ${ }^{\text {hi }} \mathrm{CD}^{+} \mathrm{T}$ cells increases in the peripheral blood and tumor tissues of cancer patients treated with anti-CTLA-4 antibodies, and the ICOS ${ }^{+}$population comprises the majority of tumor-specific, IFN- $\gamma$-producing $\mathrm{CD}^{+} \mathrm{T}$ cells $(33,34)$. Here, we have further demonstrated that redirection of $\mathrm{CD}^{+} \mathrm{T}$ cells with an ICOS-based CAR enhances the persistence of $\mathrm{CD} 8^{+} \mathrm{T}$ cells redirected with CD28or 4-1BB-based CARs, revealing the importance of proper redirection of $\mathrm{CD} 4^{+} \mathrm{T}$ cells. The role of $\mathrm{CD} 4^{+} \mathrm{T}$ cells in enhancing the persistence of $\mathrm{CD}^{+}$has been previously suggested in patients treated with adoptive cell transfer. In a trial using GD2-CAR T cells in patients with neuroblastoma, an extremely strong correlation was found between the proportion of $\mathrm{CD}^{+} \mathrm{T}$ cells and $\mathrm{CD} 45 \mathrm{RO}^{+} \mathrm{CD}_{2} 2 \mathrm{~L}^{+}$central memory cells present in infused $\mathrm{T}$ cells and the subsequent blood persistence of CAR T cells in patients (11). Similarly, adoptive transfer of tumor-infiltrating lymphocytes to patients with metastatic melanoma showed an increase in 
objective responses and $\mathrm{T}$ cell persistence in patients who received tumor-infiltrating lymphocytes containing $\mathrm{CD}^{+} \mathrm{T}$ cells compared with $\mathrm{CD} 8^{+} \mathrm{T}$ cell clones (35).

ICOS costimulation has also been shown to enhance antitumor activity mediated by $\mathrm{CD} 8^{+}$effector cells in the absence of $\mathrm{CD}^{+} \mathrm{T}$ cell help (36). We found that signaling through an ICOS-based CAR increased the cytokine release and in vivo persistence of $\mathrm{CD} 8^{+} \mathrm{T}$ cells when compared with first-generation CARs. However, the persistence of $\mathrm{CD}^{+} \mathrm{T}$ cells was further increased when $\mathrm{CD}^{+} \mathrm{T}$ cells were redirected with a 4-1BB-based CAR. It has been demonstrated previously that endogenous 4-1BB signaling affects the size, quality, and maintenance of the memory $\mathrm{CD}^{+} \mathrm{T}$ cell pool $(28,37) .4-1 \mathrm{BB}$ stimulation can rescue $\mathrm{T}$ cells from anergy and exhaustion $(25,38)$, and $4-1 \mathrm{BB}$ has been implicated in imparting long-term survival benefits to T cells (39). CAR T cells containing the 4-1BB ICD have induced complete responses in patients with $\mathrm{B}$ cell malignancies and have shown long-term survival and sustained effector functions $(6,40)$. Interestingly, we recently reported that 4-1BB signaling enhances respiratory capacity and mitochondrial biogenesis in CAR $\mathrm{T}$ cells and is associated with the expansion of a $\mathrm{CD} 8^{+}$central memory population, which may explain - in part - the differential persistence observed in clinical trials (41). Here, we provide evidence that it is possible to further increase the persistence of CAR $\mathrm{T}$ cells redirected with $4-1 \mathrm{BB}$ by combining 4-1BB and ICOS signaling domains in a third-generation CAR.

A previous report documented that constructs in which CD28 is placed distal to the cell membrane and $\mathrm{CD} 3 \zeta$ is moved to a proximal-distal location are nonfunctional (42). Most of the third-generation CARs reported, to date, combine CD28 and 4-1BB or CD28 and OX40, with CD28 placed proximal to the cell membrane (43-45). While it has been clearly demonstrated that second-generation CARs outperform first-generation CARs both in preclinical studies and clinical trials $(14,46)$, the degree to which inclusion of additional costimulatory domains increases efficacy remains unclear. Third-generation CARs have shown similar or slightly improved effector functions compared with second-generation CARs, although cytokine production levels vary in comparison with second-generation CARs, with both increased and decreased production levels reported. Initial attempts to increase $\mathrm{T}$ cell persistence in vivo with third-generation CARs have focused primarily on combined CD28 and 4-1BB signaling. Using a different strategy, Zhao et al. examined the effects of using actual ligands for different costimulatory receptors to identify the optimal combination of signals to support the persistence and function of engineered CD19 CAR T cells (47). Here, we found that combination of ICOS and 4-1BB in a third-generation CAR showed enhanced antitumor effects and synergistic effects on $\mathrm{T}$ cell in vivo survival but only when ICOS is placed proximal to the membrane. Collectively, these results support the importance of optimizing costimulation on CAR T cells.

ICOS and CD28, both members of the CD28 family, have unique and overlapping functions that synergize to regulate $\mathrm{T}$ cell differentiation. Here, we show that both $\mathrm{CD} 4^{+}$and $\mathrm{CD} 8^{+} \mathrm{T}$ cells expressing an ICOS-based CAR persisted significantly better than T cells expressing a CD28-based CAR. Several studies have shown that ICOS activates PI3K signaling more potently than CD28 $(24,48)$, resulting in increased Akt signaling. Here, we show that signaling through ICOS-based second-generation CARs induced Akt phosphorylation to greater levels than CD28-based second-generation CARs. The potent activation of Akt may explain, in part, the enhanced T cell persistence of ICOS-based CAR T cells, as Akt is a known T cell survival factor. Although PI3K is the main signaling molecule that is known to interact with ICOS, it has been recently reported that the cytoplasmic tail of ICOS has 2 potentially novel evolutionary conserved motifs (49). The ICOS proximal motif is homologous to a conserved motif found in the TNFR-associated factors TRAF2 and TRAF3, and it binds to the kinase TBK1 (49). How these ICOS-specific motifs contribute to the observed phenotype of ICOS-based CAR T cells is currently under investigation.

Some CAR T cells can have antigen-independent tonic signaling during ex vivo expansion, which can induce $\mathrm{T}$ cell differentiation and exhaustion. Tonic signaling may result when CARs are highly expressed from strong promoters in viral vectors, leading to clustering of CAR molecules. CARs with constitutive signaling have been associated with inferior antitumor activity in vivo $(26,50,51)$, highlighting the advantage of CARs whose configuration does not induce tonic signaling. Our results show that ICOS-based second-generation CARs and third-generation CARs in which ICOS is proximal to the cell membrane fail to induce tonic signaling. In contrast, CARs containing membrane-proximal 4-1BB ICD displayed tonic signaling, accompanied by accelerated $\mathrm{T}$ cell differentiation and exhaustion in the absence of cognate antigen. Different strategies have been proposed to reduce tonic signaling, including 
use of the 4-1BB ICD (25), optimization of the spacer length (50), targeting of a CAR to the T cell receptor $\alpha$ constant TRAC locus (52), or use of self-inactivating LVs instead of gammaretroviral vectors (51). While Long et al. showed that in a CAR targeting GD2 tonic signaling could be averted by substituting the CD28 ICD by the 4-1BB (25), a recent study suggested that tonic signaling observed in 4-1BB-based CARs can induce $\mathrm{T}$ cell apoptosis via continuous TRAF2-dependent activation of the NFkB pathway and FAS-dependent cell death (51). Here, we propose that, by placing the 4-1BB intracellular domain distal to the cell membrane in a ICOSBBz third-generation CAR, we can reduce tonic signaling and increase antitumor efficacy and $\mathrm{T}$ cell persistence.

Although initially considered to be an inert link between the extracellular domains and ICDs of the CAR, it is becoming clear that the TM domain may be important (53). For example, it has been previously shown that the CD3 $\zeta$ TM domain is necessary for signaling by a first-generation CAR targeting carcinoembryonic antigen (54). It has also been demonstrated that CARs incorporating the native CD3 $\zeta$ TM domain form a complex with the endogenous TCR/CD3 complex, resulting in optimal T cell acti-

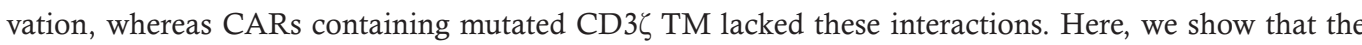
ICOS TM domain is required for the optimal in vivo phenotype of the ICOSBBz CAR T cells. We found that the ICOS TM domain does not have a key role in CAR cell-surface expression or mitigation of tonic signaling. Whether the ICOS TM is interacting with elements of the TCR complex or other endogenous molecules that enhance $\mathrm{T}$ function is an interesting question for future investigation.

Therapeutic treatments should balance efficacy and toxicity. However, the lack of good animal models has limited the evaluation of the toxicity of ICOSBBz CAR T cells. Different centers performing clinical trials with CAR $\mathrm{T}$ cells for the treatment of hematologic malignancies have reported toxicities, including cytokine release syndrome, tumor lysis syndrome, or neurologic toxicity $(2,5,6,55)$. These toxicities usually result from in vivo CAR T cell proliferation after antigen recognition. In our clinical trial testing SS1-BBz CAR T cells for the treatment of solid tumors expressing mesothelin, we observed poor $\mathrm{T}$ cell persistence and minimal efficacy with no signs of toxicity. These results correlate with results observed in other clinical trials testing CAR T cells against solid tumors in which disappointing clinical trial outcomes were also associated with poor $\mathrm{T}$ cell persistence $(8,56,57)$. Therefore, the goal of the current study was to increase $\mathrm{T}$ cell persistence to achieve improved antitumor effects. We are currently investigating different strategies to attenuate activity of CAR T cells against normal tissues that may express the target antigen, like introduction of a safety off-switch, which may help decrease the risk/benefit ratio of our ICOSBBz CAR T cells.

In summary, we demonstrate that the selection of specific costimulatory domains to redirect $\mathrm{CD} 4^{+}$ and $\mathrm{CD}^{+} \mathrm{CAR} \mathrm{T}$ cells, as well as the configuration of scFv, TM domain, and intracellular domains, are essential to optimize CAR T cell function. These results provide important information that should inform the design of next-generation cellular therapies with enhanced function and persistence, and they provide a strong rationale to test ICOSBBz-based CAR T cells in human trials.

\section{Methods}

Cell lines. All cell lines were obtained from American Type Culture Collection (ATCC), with the exception of L55 (non-small cell lung cancer), which was derived from a patient's tumor at the University of Pennsylvania. The human cancer cell lines SKOV3 (ovarian cystadenocarcinoma), L55, and K562 (myelogenous leukemia) were cultured in RPMI-1640. The Capan-2 (pancreatic adenocarcinoma) and ASPC-1 (pancreatic adenocarcinoma ascites metastasis) cell lines were cultured in DMEM/F12. All media was supplemented with 10\% FCS. All tumor cell lines were regularly validated to be Mycoplasma free. SKOV3, Capan-2, ASPC1, and K562 cells were authenticated in 2016 by the University of Arizona Genetics Core (Tucson, Arizona, USA).

Vector design. LVs expressing EF-1 $\alpha$ promoters and CARs SS1- $\delta-z$, SS1-z, SS1-BBz, SS1-28z, and SS1-ICOSz have been previously described $(21,43)$, as have LVs with truncated PGK promoters (26). Chimeric cDNA sequences containing TM ICOS-ICOS-BB-CD3z, TM CD8 $\alpha-I C O S-41 B B-C D 3 z$, and TM CD8 $\alpha-41 B B-I C O S-C D 3 z$ were custom synthesized (Integrated DNA Technologies), digested with NaeI and SalI and ligated into the pTRPE LV containing SS1-ICOSz digested with NaeI and SalI.

Isolation, transduction, and expansion of primary human t lymphocytes. Blood samples were obtained from the Human Immunology Core at the University of Pennsylvania. Peripheral blood $\mathrm{CD}^{+}$and $\mathrm{CD}^{+} \mathrm{T}$ cells were negatively isolated using RosetteSep Kits (Stem Cell Technologies). T cells were stimulated with antiCD3/CD28 magnetic beads (Invitrogen) and transduced by LVs at an MOI of 5, as previously described. For $\mathrm{CD}^{+} \mathrm{T}$ cells, human IL-2 (Prometheus) was added every other day to a final concentration of $50 \mathrm{IU} /$ 
ml. CAR T cell preparations were mixed to achieve a $1: 1 \mathrm{CD} 4^{+} / \mathrm{CD} 8^{+}$cell ratio and normalized to $50 \%$ CAR expression before functional evaluation.

In vitro coculture experiments. Tumor cells $\left(1 \times 10^{5}\right.$ cells $)$ were seeded in 48 -well plates and, after 24 hours, $3 \times 10^{5} \mathrm{~T}$ cells were added (effector/target ratio [E:T] of 3). For assays using artificial APC (aAPC), $2 \times 10^{5}$ mesothelin-expressing K562 (K562meso) cells were mixed with $4 \times 10^{5} \mathrm{~T}$ cells in 48-well plates (E:T of 2). For cytokine production assays, supernatants were collected 24 hours after coculture and analyzed using the human cytokine 30-plex panel on the Luminex system (ThermoFisher Scientific) as previously described or assessed for human IL-2, TNF- $\alpha$, and IFN- $\gamma$ using the DuoSet ELISA Development Kit (R\&D Systems). For T cell proliferation assays, cells were collected before coculture and after 8 days of incubation with K562meso cells and were stained for viability followed by surface staining for CD45, CD4, CD8, and CARs. Absolute T cell counts were determined using CountBright fluorescent beads (Invitrogen)

Flow cytometry. For all experiments, T cell suspensions were stained with a fixable live/dead violet stain (L34955, Invitrogen) in PBS followed by surface antibody staining in FACS buffer. The Foxp3/ Transcription Factor Staining Buffer set (Invitrogen) was used for Ki67 detection. The following antibodies were used: anti-CD45-PerCp-Cy5.5 (catalog 45-9459-42), anti-CD8-APC (catalog 17-0086-42), anti-CD4-PE (catalog 12-0048-42), anti-TIM3-PerCPeF710 (catalog 46-3109-42), anti-CD200-PECy7 (catalog 25-9200-42), anti-CD25-PECy7 (catalog 25-0257-42), and anti-Ki67-FITC (11-5699-42) (all from Invitrogen); anti-CD8-APC-H7 (catalog 560179) and anti-CCR7-FITC (catalog 150503) (both from BD biosciences); anti-CD4-BV510 (catalog 317444) and anti-PD-1-BV711 (catalog 329928) (both from BioLegend); and anti-CD27-PECy7 (catalog A54823) and anti-CD28-PE-CF594 (catalog 6607111) (both from Beckman Coulter). Expression of CAR proteins was evaluated using biotinylated goat anti-mouse IgG (115-065-072, Jackson ImmunoResearch) with streptavidin (APC or PE) (BD Biosciences). All experiments were conducted on a BD Fortessa flow cytometer (BD Biosciences) and analyzed with FlowJo software (Tree Star Inc.).

Western blot. T cells $\left(1 \times 10^{6}\right.$ cells $)$ were stimulated with magnetic beads coated with a yeast-derived recombinant mesothelin (58). Cell pellets were collected and frozen at indicated time points after antigen recognition. Protein lysates from cells lines were prepared in a lysis buffer containing 2\% SDS (MilliporeSigma), 62.5 mM Tris- $\mathrm{HCl}$ (MilliporeSigma), pH 6.8, 10\% glycerol (Invitrogen), 5\% 2-mercaptoethanol (Bio-Rad), and $0.002 \%$ bromophenol blue (Bio-Rad). Equivalent amounts of protein were resolved by SDS-PAGE and processed by immunoblotting analysis. The following primary antibodies were used: anti-phospho-Erk1/2 (catalog 9106), anti-Erk1/2 (catalog 9102), anti-phospho-Akt (catalog 4051), and anti-Akt (catalog 11E7) (all from Cell Signaling Technology) and anti-CD3 (catalog 551033, $\mathrm{BD}$ Biosciences). Bands were visualized by enhanced chemiluminescence (ECL). Images were captured using an Odyssey Fc system (Li-Cor Biosciences). Image processing and densitometry analysis were carried out using the Image Studio Lite software (Li-Cor Biosciences).

Mice. NSG mice were purchased from The Jackson Laboratory and bred and housed in the vivarium at the University of Pennsylvania in pathogen-free conditions.

In vivo xenograft studies. Xenograft tumors were established by s.c. injection of $5 \times 10^{6}$ L55, SKOV3, or Capan-2 cells in the presence of a $50 \%$ solution of Matrigel (BD Biosciences) in PBS. After mean tumor volume reached $200-250 \mathrm{~mm}^{3}$, mice were treated with 2 i.v. injections of $1 \times 10^{7}$ CART cells $\left(50 \% \mathrm{CAR}^{+}, 1: 1 \mathrm{CD} 4 / \mathrm{CD} 8\right.$ ratio) on days 0 and 5 . Tumor dimensions were measured with calipers, and tumor volumes calculated using the formula $\mathrm{V}=1 / 2 \times \mathrm{L} \times \mathrm{W} \times \mathrm{W}$, where $\mathrm{L}$ is length and $\mathrm{W}$ is width. For survival studies, the endpoint was established at tumor volume $\geq 1,000 \mathrm{~mm}^{3}$. Peripheral blood was obtained from retro-orbital bleeding and stained for the presence of human CD45, CD4, and CD8 T cells. After gating on the human $\mathrm{CD} 45^{+}$population, the $\mathrm{CD} 4^{+}$and $\mathrm{CD} 8^{+}$subsets were quantified using TruCount tubes (BD Biosciences). At the endpoint, spleens were harvested and filtered through 100- $\mu \mathrm{m}$ nylon mess cell strainers. RBC were lysed, and the cell suspension was filtered again through $70-\mu \mathrm{m}$ cell strainers. Intrasplenic human $\mathrm{T}$ cells were isolated using the mouse Lineage Cell Depletion Kit (Miltenyi Biotec). All experiments were performed in a blinded, randomized fashion.

Ex vivo analysis of splenocytes. Isolated human $\mathrm{T}$ cells from mouse spleens were stained for human CD45, CD4, CD8, and CAR expression. To analyze $\mathrm{T}$ cell function, isolated $\mathrm{T}$ cells were cocultured with K562meso (2:1 E:T ratio), and supernatants were collected 24 hours later. Cytokines were analyzed using the human cytokine 30-plex panel on the Luminex system (ThermoFisher Scientific). 
Statistics. All statistical analysis was performed using GraphPad Prism v6 (GraphPad Software Inc.). For comparisons of 2 groups, 2-tailed unpaired $\underline{t}$ tests were used. One-way ANOVA with Tukey post hoc test was used for comparison of 3 or more groups in a single condition. Statistical analysis for tumor volume was performed using 2-way repeated-measures ANOVA. Kaplan-Meier survival data were analyzed using a log rank (Mantel-Cox) test. Correlation was estimated by calculation of 2-tailed Pearson coefficients and significance. Data was transformed when needed to normalize variance. Symbols indicate statistical significance as follows: ${ }^{*} P<0.05 ;{ }^{*} P<0.01$, and ${ }^{* *} P<0.001$.

Study approval. The University of Pennsylvania IACUC approved all animal experiments.

\section{Author contributions}

SG, ADP, and CHJ designed the study and planned the experiments. SG, ADP, CS, AW, PRP, SEM, OUK, VCM, and MUH performed the experiments. SG, PRP, VCM, and AW analyzed the data. ADP, DS, and JS generated plasmids. SFL and JJM performed the Luminex assays. TD and CS performed the animal experiments. SG and BK wrote the manuscript. BK, ADP, SEM, RMY, and CHJ edited the manuscript.

\section{Acknowledgments}

The authors thank the Perelman School of Medicine Stem Cell \& Xenograft Core and the Human Immunology Core. The authors would like to thank Fang Chen and Natalka Koterba for the support with the Luminex cytokine assays and Edward Pequignot for help with statistics. This project was supported by a sponsored research grant from Novartis. This project was supported by a sponsored research grant from Novartis and NIH 5R01CA120409 to CHJ.

Address correspondence to: Carl H. June or Sonia Guedan, 3400 Civic Center Boulevard, 8th Floor, Room 08-123 (C.H. June), Room 08-175 (S. Guedan), Philadelphia, Pennsylvania 19104-5156, USA. Phone: 215.573.3269; Fax: Email: cjune@upenn.edu (C.H. June). Phone: 215.573.4187; Email: sgued@upenn.edu (S. Guedan).

1. Gill S, Maus MV, Porter DL. Chimeric antigen receptor T cell therapy: 25years in the making. Blood Rev. 2016;30(3):157-167.

2. Kochenderfer JN, et al. B-cell depletion and remissions of malignancy along with cytokine-associated toxicity in a clinical trial of anti-CD19 chimeric-antigen-receptor-transduced T cells. Blood. 2012;119(12):2709-2720.

3. Brentjens RJ, et al. CD19-targeted T cells rapidly induce molecular remissions in adults with chemotherapy-refractory acute lymphoblastic leukemia. Sci Transl Med. 2013;5(177):177ra38.

4. Porter DL, Levine BL, Kalos M, Bagg A, June CH. Chimeric antigen receptor-modified T cells in chronic lymphoid leukemia. N Engl J Med. 2011;365(8):725-733.

5. Grupp SA, et al. Chimeric antigen receptor-modified T cells for acute lymphoid leukemia. N Engl J Med. 2013;368(16):1509-1518.

6. Maude SL, et al. Chimeric antigen receptor T cells for sustained remissions in leukemia. N Engl J Med. 2014;371(16):1507-1517.

7. Turtle CJ, et al. CD19 CAR-T cells of defined CD4+:CD8+ composition in adult B cell ALL patients. J Clin Invest. 2016;126(6):2123-2138.

8. Kershaw MH, et al. A phase I study on adoptive immunotherapy using gene-modified T cells for ovarian cancer. Clin Cancer Res. 2006;12(20 Pt 1):6106-6115.

9. Lamers $\mathrm{CH}$, et al. Treatment of metastatic renal cell carcinoma with autologous T-lymphocytes genetically retargeted against carbonic anhydrase IX: first clinical experience. J Clin Oncol. 2006;24(13):e20-e22.

10. Ahmed N, et al. Human Epidermal Growth Factor Receptor 2 (HER2)-Specific Chimeric Antigen Receptor-Modified T Cells for the Immunotherapy of HER2-Positive Sarcoma. J Clin Oncol. 2015;33(15):1688-1696.

11. Louis CU, et al. Antitumor activity and long-term fate of chimeric antigen receptor-positive $\mathrm{T}$ cells in patients with neuroblastoma. Blood. 2011;118(23):6050-6056

12. Jensen MC, et al. Antitransgene rejection responses contribute to attenuated persistence of adoptively transferred CD20/ CD19-specific chimeric antigen receptor redirected T cells in humans. Biol Blood Marrow Transplant. 2010;16(9):1245-1256.

13. Lamers $\mathrm{CH}$, et al. Parallel detection of transduced $\mathrm{T}$ lymphocytes after immunogene therapy of renal cell cancer by flow cytometry and real-time polymerase chain reaction: implications for loss of transgene expression. Hum Gene Ther. 2005;16(12):1452-1462.

14. van der Stegen SJ, Hamieh M, Sadelain M. The pharmacology of second-generation chimeric antigen receptors. Nat Rev Drug Discov. 2015;14(7):499-509.

15. Sadelain M, Brentjens R, Rivière I. The basic principles of chimeric antigen receptor design. Cancer Discov. 2013;3(4):388-398

16. Muranski P, et al. Th17 cells are long lived and retain a stem cell-like molecular signature. Immunity. 2011;35(6):972-985.

17. Gattinoni L, et al. A human memory T cell subset with stem cell-like properties. Nat Med. 2011;17(10):1290-1297.

18. Sun JC, Williams MA, Bevan MJ. CD4+ T cells are required for the maintenance, not programming, of memory CD8+ T cells after acute infection. Nat Immunol. 2004;5(9):927-933.

19. Sun JC, Bevan MJ. Defective CD8 T cell memory following acute infection without CD4 T cell help. Science. 2003;300(5617):339-342.

20. Shedlock DJ, Shen H. Requirement for CD4 T cell help in generating functional CD8 T cell memory. Science. 
2003;300(5617):337-339.

21. Guedan S, et al. ICOS-based chimeric antigen receptors program bipolar TH17/TH1 cells. Blood. 2014;124(7):1070-1080.

22. Muranski P, Restifo NP. Adoptive immunotherapy of cancer using CD4(+) T cells. Curr Opin Immunol. 2009;21(2):200-208.

23. Hutloff A, et al. ICOS is an inducible T-cell co-stimulator structurally and functionally related to CD28. Nature. 1999;397(6716):263-266.

24. Fos C, et al. ICOS ligation recruits the p50alpha PI3K regulatory subunit to the immunological synapse. J Immunol. 2008;181(3):1969-1977.

25. Long AH, et al. 4-1BB costimulation ameliorates T cell exhaustion induced by tonic signaling of chimeric antigen receptors. Nat Med. 2015;21(6):581-590.

26. Frigault MJ, et al. Identification of chimeric antigen receptors that mediate constitutive or inducible proliferation of $\mathrm{T}$ cells. Cancer Immunol Res. 2015;3(4):356-367.

27. Abe R, Vandenberghe P, Craighead N, Smoot DS, Lee KP, June CH. Distinct signal transduction in mouse CD4+ and CD8+ splenic T cells after CD28 receptor ligation. J Immunol. 1995;154(3):985-997.

28. Shuford WW, et al. 4-1BB costimulatory signals preferentially induce CD8+ T cell proliferation and lead to the amplification in vivo of cytotoxic T cell responses. J Exp Med. 1997;186(1):47-55.

29. Maus MV, et al. Ex vivo expansion of polyclonal and antigen-specific cytotoxic T lymphocytes by artificial APCs expressing ligands for the T-cell receptor, CD28 and 4-1BB. Nat Biotechnol. 2002;20(2):143-148.

30. Milone MC, et al. Chimeric receptors containing CD137 signal transduction domains mediate enhanced survival of T cells and increased antileukemic efficacy in vivo. Mol Ther. 2009;17(8):1453-1464.

31. Teachey DT, et al. Identification of Predictive Biomarkers for Cytokine Release Syndrome after Chimeric Antigen Receptor T-cell Therapy for Acute Lymphoblastic Leukemia. Cancer Discov. 2016;6(6):664-679.

32. Takahashi N, et al. Impaired CD4 and CD8 effector function and decreased memory T cell populations in ICOS-deficient patients. J Immunol. 2009;182(9):5515-5527.

33. Ng Tang D, et al. Increased frequency of ICOS+ CD4 T cells as a pharmacodynamic biomarker for anti-CTLA-4 therapy. Cancer Immunol Res. 2013;1(4):229-234.

34. Liakou CI, et al. CTLA-4 blockade increases IFNgamma-producing CD4+ICOShi cells to shift the ratio of effector to regulatory T cells in cancer patients. Proc Natl Acad Sci USA. 2008;105(39):14987-14992.

35. Dudley ME, et al. Cancer regression and autoimmunity in patients after clonal repopulation with antitumor lymphocytes. Science. 2002;298(5594):850-854

36. Wallin JJ, Liang L, Bakardjiev A, Sha WC. Enhancement of CD8+ T cell responses by ICOS/B7h costimulation. J Immunol. 2001;167(1):132-139.

37. Hendriks J, et al. During viral infection of the respiratory tract, CD27, 4-1BB, and OX40 collectively determine formation of CD8+ memory T cells and their capacity for secondary expansion. J Immunol. 2005;175(3):1665-1676.

38. Habib-Agahi M, Phan TT, Searle PF. Co-stimulation with 4-1BB ligand allows extended T-cell proliferation, synergizes with CD80/CD86 and can reactivate anergic T cells. Int Immunol. 2007;19(12):1383-1394.

39. Sabbagh L, Pulle G, Liu Y, Tsitsikov EN, Watts TH. ERK-dependent Bim modulation downstream of the 4-1BB-TRAF1 signaling axis is a critical mediator of CD8 T cell survival in vivo. J Immunol. 2008;180(12):8093-8101.

40. Porter DL, et al. Chimeric antigen receptor T cells persist and induce sustained remissions in relapsed refractory chronic lymphocytic leukemia. Sci Transl Med. 2015;7(303):303ra139.

41. Kawalekar OU, et al. Distinct Signaling of Coreceptors Regulates Specific Metabolism Pathways and Impacts Memory Development in CAR T Cells. Immunity. 2016;44(2):380-390.

42. Finney HM, Lawson AD, Bebbington CR, Weir AN. Chimeric receptors providing both primary and costimulatory signaling in T cells from a single gene product. J Immunol. 1998;161(6):2791-2797.

43. Carpenito $\mathrm{C}$, et al. Control of large, established tumor xenografts with genetically retargeted human T cells containing CD28 and CD137 domains. Proc Natl Acad Sci USA. 2009;106(9):3360-3365.

44. Zhong XS, Matsushita M, Plotkin J, Riviere I, Sadelain M. Chimeric antigen receptors combining 4-1BB and CD28 signaling domains augment PI3kinase/AKT/Bcl-XL activation and CD8+ T cell-mediated tumor eradication. Mol Ther. 2010;18(2):413-420.

45. Pulè MA, Straathof KC, Dotti G, Heslop HE, Rooney CM, Brenner MK. A chimeric T cell antigen receptor that augments cytokine release and supports clonal expansion of primary human T cells. Mol Ther. 2005;12(5):933-941.

46. Savoldo B, et al. CD28 costimulation improves expansion and persistence of chimeric antigen receptor-modified T cells in lymphoma patients. J Clin Invest. 2011;121(5):1822-1826.

47. Zhao Z, et al. Structural Design of Engineered Costimulation Determines Tumor Rejection Kinetics and Persistence of CAR T Cells. Cancer Cell. 2015;28(4):415-428.

48. Parry RV, Rumbley CA, Vandenberghe LH, June CH, Riley JL. CD28 and inducible costimulatory protein Src homology 2 binding domains show distinct regulation of phosphatidylinositol 3-kinase, Bcl-xL, and IL-2 expression in primary human CD4 T lymphocytes. J Immunol. 2003;171(1):166-174.

49. Pedros C, et al. A TRAF-like motif of the inducible costimulator ICOS controls development of germinal center TFH cells via the kinase TBK1. Nat Immunol. 2016;17(7):825-833.

50. Watanabe N, et al. Fine-tuning the CAR spacer improves T-cell potency. Oncoimmunology. 2016;5(12):e1253656.

51. Gomes-Silva D, et al. Tonic 4-1BB Costimulation in Chimeric Antigen Receptors Impedes T Cell Survival and Is Vector-Dependent. Cell Rep. 2017;21(1):17-26.

52. Eyquem J, et al. Targeting a CAR to the TRAC locus with CRISPR/Cas9 enhances tumour rejection. Nature. 2017;543(7643):113-117.

53. Alabanza L, et al. Function of Novel Anti-CD19 Chimeric Antigen Receptors with Human Variable Regions Is Affected by Hinge and Transmembrane Domains. Mol Ther. 2017;25(11):2452-2465.

54. Bridgeman JS, Hawkins RE, Bagley S, Blaylock M, Holland M, Gilham DE. The optimal antigen response of chimeric antigen receptors harboring the $\mathrm{CD} 3 z$ zeta transmembrane domain is dependent upon incorporation of the receptor into the endogenous TCR/CD3 complex. J Immunol. 2010;184(12):6938-6949. 
55. Davila ML, et al. Efficacy and toxicity management of 19-28z CAR T cell therapy in B cell acute lymphoblastic leukemia. Sci Transl Med. 2014;6(224):224ra25.

56. Brown CE, et al. Bioactivity and Safety of IL13R 22 -Redirected Chimeric Antigen Receptor CD8+ T Cells in Patients with Recurrent Glioblastoma. Clin Cancer Res. 2015;21(18):4062-4072.

57. Park JR, et al. Adoptive transfer of chimeric antigen receptor re-directed cytolytic T lymphocyte clones in patients with neuroblastoma. Mol Ther. 2007;15(4):825-833.

58. Bergan L, Gross JA, Nevin B, Urban N, Scholler N. Development and in vitro validation of anti-mesothelin biobodies that prevent CA125/Mesothelin-dependent cell attachment. Cancer Lett. 2007;255(2):263-274. 